\title{
One-pot conversion of furfural to useful bio-products in the presence of a Sn,Al-containing zeolite beta catalyst prepared via
}

\section{post-synthesis routes}

Margarida M. Antunes ${ }^{a}$, Sérgio Lima ${ }^{b}$, Patrícia Neves ${ }^{a}$, Ana L. Magalhães ${ }^{a}$, Enza Fazio ${ }^{c}$, Auguste Fernandes ${ }^{\mathrm{d}}$, Fortunato Neri ${ }^{\mathrm{c}}$, Carlos M. Silva ${ }^{\mathrm{a}}$, Silvia M. Rocha ${ }^{\mathrm{e}}$, Maria F. Ribeiro ${ }^{\mathrm{d}}$, Martyn Pillinger ${ }^{\mathrm{a}}$, Atsushi Urakawa ${ }^{\mathrm{b}}$, Anabela A. Valente ${ }^{\mathrm{a} * *}$

a CICECO, Department of Chemistry, University of Aveiro, Campus Universitário de Santiago, 3810-193 Aveiro, Portugal

${ }^{\mathrm{b}}$ Institute of Chemical Research of Catalonia, (ICIQ), Av. Països Catalans 16, 43007 Tarragona, Spain

c Dipartimento di Fisica e di Scienze della Terra, Università degli Studi di Messina, Viale F. Stagno d'Alcontres, 3198166 Messina, Italy

d Institute for Biotechnology and Bioengineering, Centre for Biological and Chemical Engineering, Instituto Superior Técnico, Av. Rovisco Pais, 1049001, Lisboa, Portugal

e Department of Chemistry, QOPNA, University of Aveiro, Campus Universitário de Santiago, 3810-193 Aveiro, Portugal

\section{ABSTRACT}

Aiming at the valorisation of furfural (Fur) via sustainable routes based on process intensification and heterogeneous catalysis, the one-pot conversion of this renewable platform chemical to useful bio-products, namely furfuryl alkyl ethers (FEs), levulinate esters (LEs), levulinic acid (LA), angelica lactones (AnLs) and $\gamma$-valerolactone (GVL), was investigated using a single heterogeneous catalyst, in 2-butanol, at $120{ }^{\circ} \mathrm{C}$. Various chemical 
reactions are involved in this process, which requires catalysts with active sites for acid and reduction chemistry. For this purpose, it was explored for the first time the catalytic potentialities of modified versions of zeolite beta containing Al and Sn sites prepared from commercially available nanocrystaline zeolite beta via post-synthesis partial dealumination followed by solid-state ion-exchange. The post-synthesis conditions influenced considerably the catalytic performances of these types of materials. The best-performing catalyst was $(\mathrm{Sn})_{\mathrm{SSIE}^{-}}$-beta1 with $\mathrm{Si} /(\mathrm{Al}+\mathrm{Sn})=19(\mathrm{Sn} / \mathrm{Al}=27.6)$, which led to total yield of bio-products of $83 \%$ at $86 \%$ Fur conversion, and exhibited steady catalytic performance for six consecutive runs. A systematic catalytic study using the prepared catalysts with different bio-products as substrates, together with the molecular level and microstructural characterisation of the materials, helped understand the effects of different material properties on the specific reaction pathways in the overall system. These studies led to mechanistic insights into the reaction network of Fur to the bio-products in alcohol media, upon which a kinetic model was developed for the first time. The superior performance of $(\mathrm{Sn})_{\mathrm{SSIE}}$-beta1 in various steps was related to the dealumination degree, dispersion and amount of Sn-sites, and acid properties.

Keywords: furfural; bio-products; zeolite beta; dealumination; solid state ion-exchange; acid catalysis; catalytic reduction

\section{Introduction}

Furfural (Fur) is a renewable platform chemical and industrially produced from hemicelluloses [1]. It can be converted to the bio-products furfuryl alcohol (FA), furfuryl alkyl ethers (FEs), levulinate esters (LEs), levulinic acid (LA), angelica lactone isomers (AnLs) and $\gamma$-valerolactone (GVL) [2-4] (Scheme 1), useful in different sectors of the chemical industry. FA, industrially produced via hydrogenation of Fur, is used in the foundry 
industry [5], and FEs are used as blending components of gasoline [6, 7] and as flavour compounds [8, 9]. LEs are used as oxygenate fuel additives [10-12], solvents, and to produce plasticizers and flavouring agents [13-14]. LA is used in the production of fuel additives [1521], agrochemicals (e.g. synthesis of $\delta$-aminolevulinic acid, a biodegradable pesticide) [20, 22], and polymers (e.g. synthesis of diphenolic acid, an alternative to bisphenol A) [20, 23]. $\alpha$-Angelica lactone ( $\alpha \mathrm{AnL})$ is used for food flavouring and as aromas in the tobacco industry [24], pheromones [25] and fuel additives [14, 26], while GVL is used as solvent for biomassrelated reactions [27, 28], chemical intermediates [16-18, 29-33] and fuels [16, 17, 34, 35]. The conversion of Fur to the bio-products is complex because it involves acid and reduction chemistry. Hence, one-pot conversion of Fur to give desired bio-products in high yields using a heterogeneous catalyst is particularly challenging.

Zeolites (crystalline microporous aluminosilicates) are versatile materials with various commercial applications, particularly as heterogeneous catalysts which led to important breakthroughs in refinery technologies. The potential application of zeolites can be extended to catalyst technology of future bio-refineries to convert biomass to fuels and chemicals, alleviating society's dependence on (non-renewable) fossil fuels [3, 36, 37]. Among the most investigated zeolites, beta with BEA framework topology possesses a 3-D large-pore channel system and 12-membered ring channels. Zeolite beta and its modified versions are known to be effective catalysts for several reactions concerning the valorisation of biomass, e.g. corn fiber to Fur [38]; levuglucosan (an intermediate of (hemi)cellulose pyrolysis) to glucose [39] or Fur [40]; saccharides to Fur [41, 42], 5-(hydroxymethyl)furfural (HMF) [42-47], or LEs [48, 49]; cellulose and hemicelluloses to diesel [50]; hemicelluloses to polyols [51]; C-3 sugar to methyl lactate and lactic acid [52]; FA to 2-(ethoxymethyl)furfural (EMF) and ethyl levulinate (EL) [53]; biodegradable surfactants via acetalisation [54] or etherification of HMF [55]; Fur to GVL [4]; pyrolysis of biomass or derived compounds to aromatic/aliphatic 
hydrocarbons [56-66]; sugarcane bagasse to bio-oil and upgrading to fuel [67]; co-conversion of biogenic waste and vegetable oil [68]; and pyrolysis of organosolv lignin to phenolic compounds $[69,70]$. The introduction of different elements into zeolite beta widens its catalytic potential. In particular, tin-containing zeolite beta (Sn-beta) can promote chemoselective reduction of carbonyl groups to alcohol groups under relatively mild conditions via the Meerwein-Ponndorf-Verley (MPV) mechanism [71], avoiding the use of high pressure $\mathrm{H}_{2}$. Quantum chemical calculations indicated that Sn-beta stands on a similar footing to the classical compound $\mathrm{Al}(\mathrm{III})$-isopropoxide used for MPV systems [72].

The method of preparing heterogeneous catalysts is an important factor from the practical point of view. The introduction of large Lewis acid centres such as $\mathrm{Sn}^{\text {IV }}$ into zeolite beta typically involves tedious hydrothermal synthesis procedures, with several limitations: long synthesis times (due to slow nucleation), reduced number of isolated metal sites introduced and formation of relatively large crystals which can lead to internal diffusion limitations during the catalytic reaction [73]. An interesting strategy to overcome these limitations is using an up-scalable simple post-synthesis protocol involving dealumination and subsequent solid-state ion-exchange (SSIE). This protocol is advantageous in comparison to conventional liquid-phase routes in that it generates less toxic waste and avoids solvation of the metal species and hydrolysis of metal precursors which can impede the incorporation of the Lewis acid centres [74]. The modification of zeolite beta via SSIE was reported in 1993 by Barthomeuf et al. [75] to introduce lanthanum, and this procedure was more efficient than classical ion-exchange in solution, without destroying the zeolitic framework. Since then, different elements have been introduced into zeolite beta by SSIE leading to catalytic performances that are superior to those reached with the corresponding materials prepared using conventional liquid-phase routes $[73,76,77]$. Successful incorporation of tin into the BEA framework by SSIE was demonstrated by Hermans et al. [73, 75]. Zeolite beta was 
partially dealuminated by acid treatment, generating vacant tetrahedral sites which were subsequently occupied by tin species introduced by SSIE.

In the present work, modified versions of zeolite beta containing aluminium and tin sites were prepared from nanocrystalline $\mathrm{NH}_{4}$-beta via post-synthesis routes similar to those described by Hermans et al. [73]. Different materials were prepared by varying the acid concentration used for the partial dealumination, the amount of tin precursor used in the SSIE process, or by carrying out $(\mathrm{Sn}, \mathrm{Al})$-competitive $\mathrm{SSIE}$. The prepared $(\mathrm{Sn}, \mathrm{Al})$-containing materials were explored as catalysts for the one-pot multistep conversion of Fur to the bioproducts, using 2-butanol $(2 \mathrm{BuOH})$ as reacting solvent, at $120^{\circ} \mathrm{C}$. In order to help understand the effects of different material properties on the specific reaction pathways in the overall reaction system, the modified beta materials were also tested as catalysts for the reactions starting from FA, FEs, LEs, $\alpha$ AnL and LA. These studies led to mechanistic insights into the complex reaction system, upon which a kinetic model was developed.

\section{Experimental}

\subsection{Preparation of modified versions of zeolite beta}

Modified versions of beta catalysts were prepared from commercial nanocrystalline $\mathrm{NH}_{4}$-beta (Zeolyst, CP814E; based on the supplier's technical information $\mathrm{Si} / \mathrm{Al}=12.5$, ca. $20-30 \mathrm{~nm}$ crystallite sizes [41]). First, $\mathrm{NH}_{4}$-beta was calcined at $550{ }^{\circ} \mathrm{C}$ for $10 \mathrm{~h}$ under static air, giving H-beta. Subsequently, H-beta was modified with $\mathrm{Sn}$ in a similar fashion to that described by

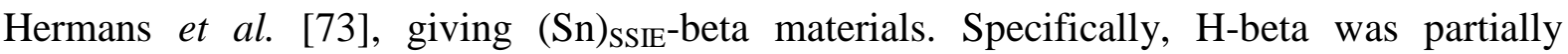
dealuminated by acid treatment at $100{ }^{\circ} \mathrm{C}$ for $20 \mathrm{~h}\left(\mathrm{HNO}_{3}(70 \%\right.$, Sigma-Aldrich) was used to prepare acid solutions with the desired concentrations). The dealuminated materials denoted as deAl-beta1, deAl-beta2 and deAl-beta3 were obtained using decreasing acid concentration (Table 1). Subsequently, these materials were subjected to SSIE with tin. Solid mixtures of 
tin(II) acetate (Sigma-Aldrich) and deAl-betan ( $n=1,2$, or 3$)$ were ground and mixed for 20 min at room temperature. After calcination at $550{ }^{\circ} \mathrm{C}$ for $4 \mathrm{~h}$, under air flow $\left(20 \mathrm{~mL} \mathrm{~min}{ }^{-1}\right)$, $(\mathrm{Sn})_{\mathrm{SSIE}^{-}}$-betan $(n=1,2$, or 3$)$ were obtained from the respective parent dealuminated materials, deAl-betan. A material denoted $(\mathrm{SnAl})_{\mathrm{SSIE}}$-beta1 was prepared from deAl-beta1 in a similar fashion to that for $(\mathrm{Sn})_{\mathrm{SSIE}^{-}}$beta1, but using an equimolar mixture of tin(II) acetate and aluminium acetylacetonate (99\%, Sigma-Aldrich) in the SSIE step prior to the calcination treatment. Bulk $\mathrm{SnO}_{2}$ was synthesised by treating tin(II) acetate under the same conditions to that used to prepare $(\mathrm{Sn})_{\mathrm{SSIE}}$-betan.

Table 1. Modification conditions and textural properties of nanocrystalline H-beta and derived materials. ${ }^{\mathrm{a}}$

\begin{tabular}{|c|c|c|c|c|c|}
\hline \multirow[b]{2}{*}{ Sample } & \multicolumn{2}{|c|}{ Post-synthesis conditions } & \multicolumn{3}{|c|}{ Textural properties } \\
\hline & $\begin{array}{c}\text { Dealumination }^{\mathrm{a}} \\
{\left[\mathrm{HNO}_{3}\right](\mathrm{M})}\end{array}$ & $\begin{array}{c}\mathrm{SSIE}^{\mathrm{b}} \\
\left(\mathrm{mmol}_{\mathrm{Sn}(\mathrm{II})+\mathrm{Al}(\mathrm{III})} / \mathrm{g}_{\text {deAl-beta }}\right)\end{array}$ & $\begin{array}{c}\mathrm{S}_{\mathrm{BET}} \\
\left(\mathrm{m}^{2} \mathrm{~g}^{-1}\right)\end{array}$ & $\begin{array}{c}S_{\text {ext }} \\
\left(\mathrm{m}^{2} \mathrm{~g}^{-1}\right)\end{array}$ & $\begin{array}{c}\mathrm{V}_{\text {micro }} \\
\left(\mathrm{cm}^{3} \mathrm{~g}^{-1}\right)\end{array}$ \\
\hline H-beta & - & - & 650 & 204 & 0.18 \\
\hline deAl-beta1 & 13 & - & 583 & 190 & 0.16 \\
\hline deAl-beta2 & 7.2 & - & 554 & 176 & 0.15 \\
\hline deAl-beta3 & 4.3 & - & 543 & 179 & 0.14 \\
\hline$(\mathrm{Sn})_{\mathrm{SSIE}}$-beta1 & 13.0 & 0.846 & 559 & 170 & 0.16 \\
\hline$(\mathrm{Sn})_{\mathrm{SSIE}}$-beta2 & 7.2 & 0.421 & 569 & 181 & 0.16 \\
\hline$(\mathrm{Sn})_{\mathrm{SSIE}}$-beta3 & 4.3 & 0.210 & 573 & 180 & 0.16 \\
\hline$(\mathrm{SnAl})_{\mathrm{SSIE}^{-} \text {-beta } 1}$ & 13.0 & $0.422(\mathrm{Sn})+0.422(\mathrm{Al})$ & 566 & 181 & 0.15 \\
\hline
\end{tabular}

\footnotetext{
${ }^{\mathrm{a}}$ In the dealumination step ( $\left.\mathrm{deAl}\right)$ the volume of the acid solution per mass of $\mathrm{H}$-beta was always $20 \mathrm{~mL} / \mathrm{g}_{\mathrm{H}-\mathrm{beta} \cdot}{ }^{\mathrm{b}}$ Amount of $\mathrm{Al}$ and/or Sn precursor used per gram of deAl-betan $(n=1,2,3)$ used in the solid-state ion-exchange (SSIE) step.
} 


\subsection{Characterisation of the catalysts}

The wide-angle XRD patterns $\left(10^{\circ}<2 \theta<70^{\circ}\right)$ were collected at room temperature on a D8 Advance Series 2 Theta/Theta powder diffraction system (Bruker) with $\mathrm{Cu}-\mathrm{K} \alpha$ radiation and step size of $0.02^{\circ}$. Scanning electron microscopy (SEM) images, energy dispersive X-ray spectroscopy (EDS) analysis and elemental (Sn, Al, Si) mappings were obtained on a Hitachi SU-70 SEM microscope with a Bruker Quantax 400 detector operating at $20 \mathrm{kV}$.

Inductively coupled plasma atomic emission spectroscopy (ICP-AES) analyses of all prepared samples were requested to the Central Analysis Laboratory (University of Aveiro); the measurements were carried out on a ICP-OES spectrometer Horiba Jobin Yvon modelo Activa M (detection limit of ca. $20 \mu \mathrm{g} \cdot \mathrm{dm}^{-3}$; experimental range of error of ca. $5 \%$ ). Prior to the ICP-AES, the solids (10 mg) were digested by microwave with $1 \mathrm{~mL}$ of $\mathrm{HF}$ and $1 \mathrm{~mL}$ of $\mathrm{HNO}_{3}$, in a closed vessel at $180{ }^{\circ} \mathrm{C}$, followed by a second digestion with $\mathrm{HCl}$.

Nitrogen and argon adsorption-desorption isotherms were measured at $-196{ }^{\circ} \mathrm{C}$ and $-186^{\circ} \mathrm{C}$, respectively, on an Autosorb-iQ (Quantochrome Instruments). Prior to measurements, the solids were out-gassed at $300{ }^{\circ} \mathrm{C}$ for $12 \mathrm{~h}$ under vacuum. From the $\mathrm{N}_{2}$ adsorption isotherms the textural properties of the materials were calculated: the specific surface area $\left(\mathrm{S}_{\mathrm{BET}}\right)$ using the Brunauer-Emmett-Teller (BET) equation, (interparticle) mesopore size ( $\left.D_{\text {meso }}\right)$ distribution using the BJH method, external surface area $\left(\mathrm{S}_{\text {ext }}\right)$ and micropore volume $\left(\mathrm{V}_{\text {micro }}\right)$ using the t-plot method. The micropore size distribution was calculated from the $\mathrm{Ar}$ adsorption isotherm using non-local DFT method (cylindrical pore model) of the ASiQwin software (version 3.01).

The ${ }^{27} \mathrm{Al}$ MAS NMR spectra of H-beta and $(\mathrm{SnAl})_{\mathrm{SSIE}}$-betal were recorded at $104.26 \mathrm{MHz}$ using a Bruker Avance $400(9.4 \mathrm{~T})$ spectrometer with a contact time of $3 \mathrm{~ms}$, a recycle delay of $1 \mathrm{~s}$, and a spinning rate of $13 \mathrm{kHz}$. For the remaining materials (deAl-beta2, $(\mathrm{Sn})_{\mathrm{SSIE}}$-beta2, 
deAl-beta1 and $(\mathrm{Sn})_{\mathrm{SSIE}}$-beta1, the ${ }^{27} \mathrm{Al}$ MAS NMR spectra were recorded at $182.432 \mathrm{MHz}$ using a Bruker Avance III HD 700 (16.4 T) spectrometer with a unique pulse, a recycle delay of $1 \mathrm{~s}$, and a spinning rate of $14 \mathrm{kHz}$. Chemical shifts are quoted in ppm from $\mathrm{Al}\left(\mathrm{NO}_{3}\right)_{3}$. Fourier transform infrared (FT-IR) spectra were recorded in transmission mode as $\mathrm{KBr}$ pellets using a Unican Mattson Mod 7000 spectrophotometer equipped with a DTGS CsI detector (400-4000 $\mathrm{cm}^{-1}, 256$ scans, $4 \mathrm{~cm}^{-1}$ resolution). Diffuse reflectance UV-vis spectra were recorded using a Jasco V-560 spectrophotometer and $\mathrm{BaSO}_{4}$ as reference. Raman measurements were carried out on a JobinYvon HR 800 UV-Raman spectrometer with the $325 \mathrm{~nm}$ He-Cd laser line. The thermogravimetric analyses (TGA) and differential scanning calorimetry (DSC) analyses were carried out under air with a heating rate of $10{ }^{\circ} \mathrm{C} \mathrm{min}^{-1}$, using Shimadzu TGA-50 and DSC-50 instruments, respectively.

X-ray photoelectron spectroscopy (XPS) analysis was performed on a K-Alpha system from Thermo Scientific, equipped with a monochromatic Al K a source (1486.6 eV), and operating in constant analyser energy (CAE) mode, with a pass energy of 200 and $50 \mathrm{eV}$ for survey and high resolution spectra, respectively. A spot size diameter of ca. $400 \mu \mathrm{m}$ was adopted. Thus the measurements were carried out over large number of randomly oriented beta type crystallites, and the results represent fairly well the average chemical environment of the samples.

The acid properties of the modified beta materials were measured using a NexusThermo Nicolet apparatus (64 scans and resolution of $4 \mathrm{~cm}^{-1}$ ) equipped with a specially designed cell, using self-supported discs $\left(5-10 \mathrm{mg} \mathrm{cm}^{-2}\right)$ and pyridine as the basic probe. Pyridine was chosen since its critical dimension of ca. $6.5 \AA$ [78] is somewhat comparable with the molecular diameters of furfural (ca. $5.7 \AA$ along the longest axis [79]). After in situ outgassing at $450{ }^{\circ} \mathrm{C}$ for $3 \mathrm{~h}\left(10^{-6}\right.$ mbar $)$, pyridine (99.99\%) was contacted with the sample at $150{ }^{\circ} \mathrm{C}$ for $10 \mathrm{~min}$ and subsequently evacuated for $30 \mathrm{~min}$ under vacuum $\left(10^{-6} \mathrm{mbar}\right)$. The IR 
bands at ca. 1540 and $1455 \mathrm{~cm}^{-1}$ are related to pyridine adsorbed on Brønsted (B) and Lewis (L) acid sites, respectively [80]. The acid properties of H-beta were considered the same as those reported by our group in ref. [41] for an identical sample, obtained from same $\mathrm{NH}_{4}$-beta recipient acquired.

\subsection{Catalytic tests}

The batch catalytic experiments were performed in tubular glass reactors with pear-shaped bottoms and equipped with an appropriate PTFE-coated magnetic stirring bar and a valve. In a typical procedure, $0.45 \mathrm{M}$ of furfural (Fur, Aldrich, 99\%) and powdered catalyst (loading of $26.7 \mathrm{~g}_{\text {cat }} \mathrm{L}^{-1}$ ) in $0.75 \mathrm{~mL}$ of aliphatic alcohol (2-butanol (Sigma-Aldrich, 99\%) or 2-propanol (Sigma-Aldrich, $\geq 99.5 \%)$ ) were added to the reactor and heated at $120{ }^{\circ} \mathrm{C}$. These reaction conditions are similar to those used by Román-Leshkov et al. [4]. Additionally, furfuryl alcohol (FA, Aldrich, 99\%), 1-butyl levulinate (1BL, Aldrich, 98\%), ethyl levulinate (EL, Aldrich, 99\%), levulinic acid (LA, Aldrich, 98\%), $\alpha$-angelica lactone ( $\alpha$ AnL, Alfa Aesar, 98\%), furfuryl 1-butyl ether (1BMF, Manchester Organics, 95\%) and furfuryl ethyl ether (EMF, Manchester Organics, 97\%) were tested as substrates.

The reaction mixtures were heated with a thermostatically controlled oil bath, under continuous magnetic stirring at $1000 \mathrm{rpm}$. Reaction time was calculated from the instant when the reactor was immersed in the oil bath. The catalytic performances of the different prepared materials were compared on the basis of similar mass of catalyst (important for practical applications).

In order to examine the recyclability of the catalyst (here only $(\mathrm{Sn})_{\mathrm{SSIE}}$-beta1 was tested), after a batch run using Fur as substrate, the solid catalyst was separated from the reaction mixture by centrifugation, thoroughly washed with 2-butanol, dried at $85{ }^{\circ} \mathrm{C}$ overnight, and calcined at $550{ }^{\circ} \mathrm{C}$ for $3 \mathrm{~h}$ with a heating rate of $1{ }^{\circ} \mathrm{C} \min ^{-1}$ to give the regenerated catalyst. 
The catalyst was used in six consecutive batch runs under similar reaction conditions. In order to check for leaching and the presence of soluble active species, contact tests were performed as follows: the catalyst was treated for $24 \mathrm{~h}$ at $120^{\circ} \mathrm{C}$ under similar conditions to those used for typical batch runs, but without substrate. Subsequently, the mixture was cooled to room temperature, the solid catalyst was separated by centrifugation, and the liquid phase was passed through a filter containing a $0.2 \mu \mathrm{m}$ PTFE membrane. The substrate was added to the obtained liquid solution to give an initial substrate concentration of $0.45 \mathrm{M}$, and this solution was stirred at $120^{\circ} \mathrm{C}$ for $24 \mathrm{~h}$.

The evolution of the catalytic reactions was monitored by GC (for quantification of the bioproducts) and HPLC (for quantification of Fur). Prior to sampling, the reactors were cooled to ambient temperature before opening and work-up procedures. The analyses were always carried out for freshly prepared samples. The GC analyses were carried out using a Varian 3800 equipped with a capillary column (Chrompack, CP-SIL 5CB, $50 \mathrm{~m} \times 0.32 \mathrm{~mm} \times 0.5$ $\mu \mathrm{m})$ and a flame ionisation detector, using $\mathrm{H}_{2}$ as carrier gas. The HPLC analyses were carried out using a KnauerSmartline HPLC Pump 100 and a Shodex SH1011 H $300 \mathrm{~mm} \times 8 \mathrm{~mm}$ (i.d.) ion exchange column (Showa Denko America, Inc., New York), coupled to a KnauerSmartline UV detector $2520(254 \mathrm{~nm})$ where the mobile phase was $0.005 \mathrm{M}$ aq. $\mathrm{H}_{2} \mathrm{SO}_{4}$ at a flow rate of $0.8 \mathrm{~mL} \min ^{-1}$ and the column temperature was $50{ }^{\circ} \mathrm{C}$. Calibration curves were measured for quantification. Individual experiments were performed for a given reaction time and the presented results are the mean values of at least two replicas. The substrate (Sub) conversion $(\%)$ at reaction time $t$ was calculated using the formula: $100 \times[$ (initial concentration of Sub)-(concentration of Sub at time $t)] /($ initial concentration of Sub). The yield of product (Pro) $(\%)$ at reaction time $t$ was calculated using the formula: $100 \times[($ concentration of Pro at time $t) /($ initial concentration of Sub) $]$. The identification of the bio-products was checked by GC-MS using a Trace GC 2000 Series (Thermo Quest CE 
Instruments)-DSQ II (Thermo Scientific), equipped with a capillary column (DB-5 MS, $30 \mathrm{~m}$ $\times 0.25 \mathrm{~mm} \times 0.25 \mu \mathrm{m})$ using $\mathrm{He}$ as carrier gas. The bio-products (whenever formed) were furfuryl alcohol (FA), furfuryl alkyl ethers (2BMF=furfuryl 2-butyl ether, 2PMF=furfuryl 2propyl ether), levulinate esters $(2 \mathrm{BL}=2$-butyl levulinate, $1 \mathrm{BL}=1$-butyl levulinate, $\mathrm{EL}=$ ethyl levulinate, $2 \mathrm{PL}=2$-propyl levulinate $)$, angelica lactones $(\alpha \mathrm{AnL}=\alpha$-angelic lactone, $\beta \mathrm{AnL}=\beta$ angelic lactone), levulinic acid (LA), and $\gamma$-valerolactone (GVL).

\subsection{Kinetic modelling}

The micro reactors were modelled as perfectly stirred batch reactors, and the mass balance equations are given by:

$$
\frac{V}{W} \frac{d C_{i}}{d t}=r_{i}
$$

where $V$ is the reaction mixture volume $(\mathrm{L}), W$ is the mass of catalyst $(\mathrm{g}), C_{\mathrm{i}}$ is the molar concentration of the reactive species $i(\mathrm{M}), t$ is time $(\mathrm{h})$, and $r_{\mathrm{i}}$ is the overall reaction rate of species $i$ expressed per unit of mass catalyst $\left(\mathrm{mol} \cdot \mathrm{g}_{\mathrm{cat}}{ }^{-1} \cdot \mathrm{h}^{-1}\right)$. The ratio $W / V$ was maintained constant in all experiments.

Based on the mechanism proposed in section 3.2.3, a pseudo-homogeneous kinetic model was developed, considering first-order reactions for all steps involved:

$$
\begin{aligned}
& \frac{V}{W} \frac{d C_{\mathrm{FUR}}}{d t}=-\left(k_{1}+k_{9}\right) C_{\mathrm{FUR}} \\
& \frac{V}{W} \frac{d C_{\mathrm{FA}}}{d t}=k_{1} C_{\mathrm{FUR}}-\left(k_{2}+k_{3}+k_{10}\right) C_{\mathrm{FA}} \\
& \frac{V}{W} \frac{d C_{2 \mathrm{BMF}}}{d t}=k_{2} C_{\mathrm{FA}}-\left(k_{4}+k_{5}+k_{11}\right) C_{2 \mathrm{BMF}} \\
& \frac{V}{W} \frac{d C_{\mathrm{AnLs}}}{d t}=k_{3} C_{\mathrm{FA}}+k_{4} C_{2 \mathrm{BMF}}-\left(k_{6}+k_{12}\right) C_{\mathrm{AnLs}}
\end{aligned}
$$




$$
\begin{aligned}
& \frac{V}{W} \frac{d C_{\mathrm{LA}}}{d t}=k_{6} C_{\mathrm{AnLs}}-\left(k_{7}+k_{13}\right) C_{\mathrm{LA}} \\
& \frac{V}{W} \frac{d C_{2 \mathrm{BL}}}{d t}=k_{5} C_{2 \mathrm{BMF}}+k_{7} C_{\mathrm{LA}}-\left(k_{8}+k_{14}\right) C_{2 \mathrm{BL}} \\
& \frac{V}{W} \frac{d C_{\mathrm{GVL}}}{d t}=k_{8} C_{2 \mathrm{BL}} \\
& \frac{V}{W} \frac{d C_{\mathrm{D}_{\mathrm{Fur}}}}{d t}=k_{9} C_{\mathrm{Fur}} \\
& \frac{V}{W} \frac{d C_{\mathrm{D}_{\mathrm{FA}}}}{d t}=k_{10} C_{\mathrm{FA}} \\
& \frac{V}{W} \frac{d C_{\mathrm{D}_{2 \mathrm{BMF}}}}{d t}=k_{11} C_{2 \mathrm{BMF}} \\
& \frac{V}{W} \frac{d C_{\mathrm{D}_{\mathrm{AnLs}}}}{d t}=k_{12} C_{\mathrm{AnLs}} \\
& \frac{V}{W} \frac{d C_{\mathrm{D}_{\mathrm{LA}}}}{d t}=k_{13} C_{\mathrm{LA}} \\
& \frac{d C_{\mathrm{D}_{2 \mathrm{BL}}}}{d t}=k_{14} C_{2 \mathrm{BL}}
\end{aligned}
$$

where $k_{j}$ are the apparent reaction kinetic constants $\left({\mathrm{L} \cdot \mathrm{g}_{\mathrm{cat}}}^{-1} \cdot \mathrm{h}^{-1}\right)$ of step $j$ at constant temperature.

The problem was solved by numerical integration with simultaneous optimization, using appropriate initial conditions (at $t=0$ ). MEIGO (MEtaheuristics for systems biology and bIoinformatics Global Optimization) [81], an open-source toolbox for global optimization, and Matlab ${ }^{\circledR}$ (version 7.8) were used to obtain the kinetic constants by fitting the model proposed to the experimental data (up to $7 \mathrm{~h}$ ) in order to minimize the following objective function:

$$
F_{\text {obj }}=\sum_{m}\left\{\sum_{n=1}^{n_{\mathrm{p}}}\left[\left.C_{m, n}\right|_{\mathrm{calc}}-\left.C_{m, n}\right|_{\mathrm{exp}}\right]^{2}\right\}
$$


where $\left.C_{m, n}\right|_{\text {calc }}$ and $\left.C_{m, n}\right|_{\exp }$ are the concentrations predicted by the model and the experimental ones, respectively, at each instant of time $n, m$ is Fur, 2BMF, AnLs, LA, 2BL or GVL.

\section{Results and Discussion}

\subsection{Characterisation of the modified zeolite beta materials}

Powder X-ray diffraction (XRD) patterns of the dealuminated materials (deAl-betan) are similar to that of H-beta (Figure 1), displaying reflections characteristic of the crystalline structure with BEA topology [41, 82-84]. The crystalline structure was preserved during the acid treatments, which is in agreement with the literature for $\mathrm{HNO}_{3}$-treated beta materials [83, 85-89]. The SSIE process did not lead to measurable changes in the crystalline structure of the materials deAl-betan. 


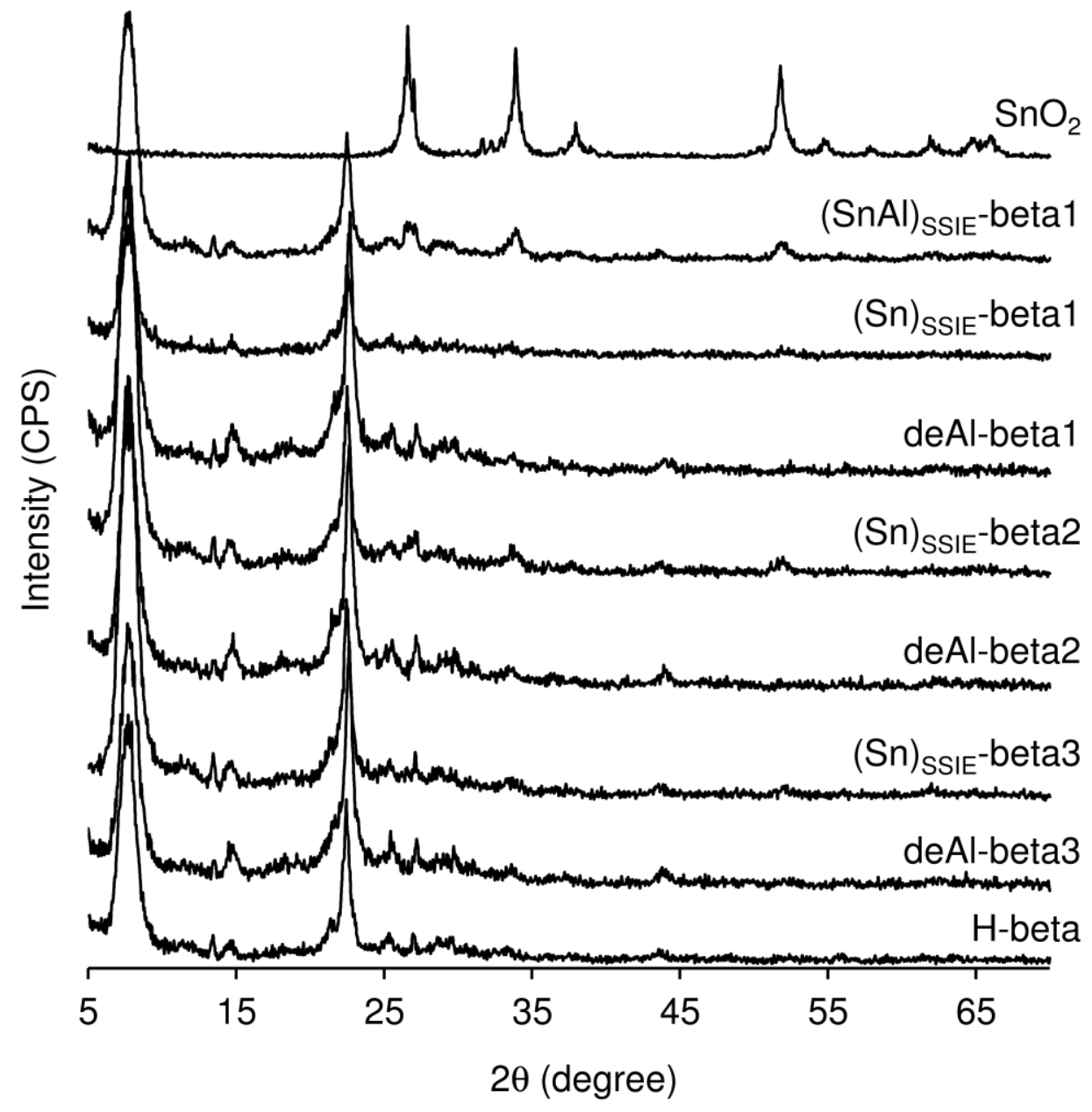

Figure 1. Powder XRD patterns of $\mathrm{H}$-beta and its modified versions, and $\mathrm{SnO}_{2}$ for comparison.

Treatment of tin(II) acetate by calcination under identical conditions to those used to prepare the $(\mathrm{Sn})_{\mathrm{SSIE}^{-}}$-betan materials after SSIE, led to the cassiterite phase of $\mathrm{SnO}_{2}($ member of the rutile group, JCPDS No. 41-1445): $2 \theta=26.6,33.9,38,52.7$ and $54.7^{\circ}$, corresponding to the reflections (100), (101), (200), (211) and (220), respectively), which consists of $\left[\mathrm{SnO}_{6}\right]^{8-}$ octahedra $[90,91]$. The bulk tin oxide sample (hereafter denoted $\mathrm{SnO}_{2}$ for the sake of simplicity) seems to be mixed with relatively small amounts of other tin oxide phases; a peak at $27^{\circ}$ may be due to $\mathrm{Sn}_{2} \mathrm{O}_{3}$ (JCPDS No. 25-1259), and a peak at $31.7^{\circ}$ may be due to triclinic $\mathrm{Sn}_{3} \mathrm{O}_{4}$ (JCPDS No. 16-757). No crystalline phases of tin oxide could be distinguished in the XRD pattern of $(\mathrm{Sn})_{\mathrm{SSIE}}$-beta1. For the remaining $\mathrm{Sn}$-containing beta materials (especially 
$(\mathrm{SnAl})_{\mathrm{SSIE}}$-beta1) a weak peak at ca. $52^{\circ}$ not related to the BEA framework structure, was observed, which may be due to crystalline $\mathrm{SnO}_{2}$ and heterogeneous dispersion of $\mathrm{Sn}$ in these materials.

Zeolite H-beta and the corresponding modified materials exhibited reversible $\mathrm{N}_{2}$ adsorptiondesorption isotherms with features of Type I. The significant increase in $\mathrm{N}_{2}$ uptake at low relative pressures $\left(\mathrm{p} / \mathrm{p}_{0}<0.1\right)$ can be attributed to the filling of micropores (Figure $\mathrm{S} 1$ ). The $\mathrm{N}_{2}$ uptake increases again significantly as $\mathrm{p} / \mathrm{p}_{0}$ approaches unity, which is likely due to multilayer adsorption on the external surface of the crystallites. The specific surface area and pore volume of H-beta decreased slightly after the acid treatments (Table 1). Comparison of the micropore size distribution of deAl-beta1 and the corresponding $(\mathrm{Sn})_{\mathrm{SSIE}}$-beta1 material showed no considerable changes in pore sizes after the SSIE (the maxima at ca. 5.8 and $6.1 \AA$ for deAl-beta1 and $(\mathrm{Sn})_{\mathrm{SSIE}}$-beta1, respectively, Figure $\left.\mathrm{S} 2\right)$. In general, the texture parameters of the modified materials are comparable $\left(\mathrm{S}_{\mathrm{BET}}\right.$ of $543-583 \mathrm{~m}^{2} \mathrm{~g}^{-1}, \mathrm{~S}_{\mathrm{ext}}$ of $170-190 \mathrm{~m}^{2} \mathrm{~g}^{-1}, \mathrm{~V}_{\text {micro }}$ of $0.14-0.16 \mathrm{~cm}^{3} \mathrm{~g}^{-1}$, Table 1$)$. The $\mathrm{S}_{\mathrm{BET}}$ values are in the range of values reported in the literature for Sn-beta materials prepared using different synthetic approaches [73, 82, 92, 93]. The materials possess considerable $S_{\text {ext }}$ and ratios $S_{\text {ext }} / V_{\text {micro }}$, which is consistent with the fact they were prepared from nano-sized crystallites of zeolite beta (ca. 20-30 nm [41]). On the other hand, the post-synthesis treatments did not cause significant structural collapse or pore blockage.

All modified beta materials consist of irregular shaped aggregates of crystallites, with homogeneous dispersions of $\mathrm{Al}$ and $\mathrm{Si}$ as observed by SEM and elemental mapping (Figures 2 and 3). The Sn mapping showed fairly homogeneous dispersion of surface species in the case of $(\mathrm{Sn})_{\mathrm{SSIE}}$-beta1, whereas for the remaining $\mathrm{Sn}$-containing beta materials, regions with higher concentrations of Sn were found, showing heterogeneous dispersion of Sn. These results are consistent with the powder XRD data of the Sn-containing solids. 


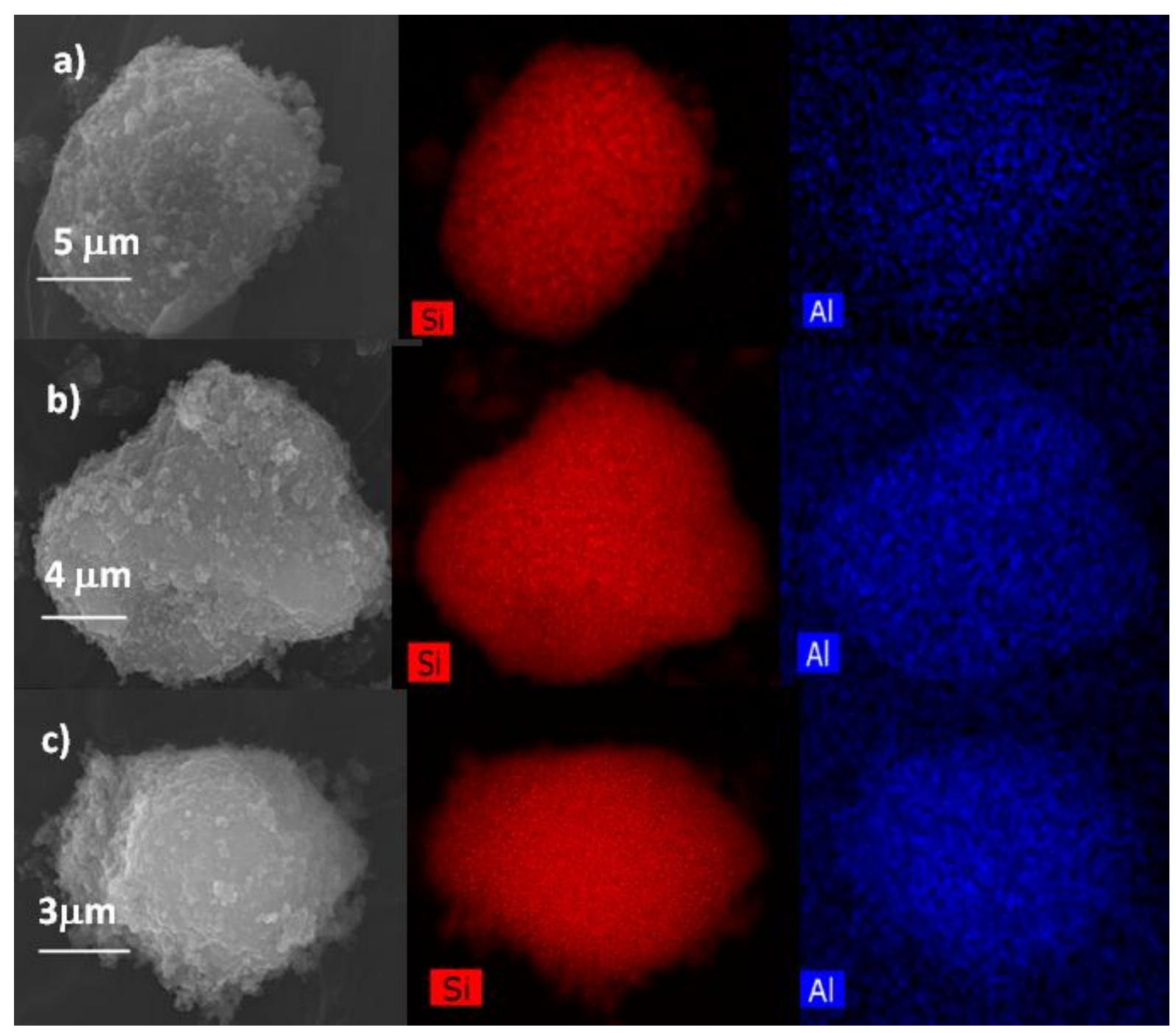

Figure 2. SEM and elemental mapping of Si and Al for deAl-beta1 (a), deAl-beta2 (b) and deAl-beta3 (c).

The compositions of the modified beta materials were determined by ICP-AES analyses (Table 2). The dealumination of $\mathrm{H}$-beta using increasingly concentrated $\mathrm{HNO}_{3}$ led increasing molar ratios $\mathrm{Si} / \mathrm{Al}$ of the materials deAl-betan, and thus the dealumination degree increased in the order, deAl-beta3<deAl-beta2<deAl-beta1. The Si/Al ratios of the dealuminated materials remained comparable after SSIE for Sn. The simultaneous increasing degree of dealumination and decreasing amount of Sn introduced by the SSIE, led to materials $(\mathrm{Sn})_{\mathrm{SSIE}^{-}}$ betan with increasing $\mathrm{Sn} / \mathrm{Al}$ and decreasing $\mathrm{Si} / \mathrm{Sn}$ ratios; $(\mathrm{Sn})_{\mathrm{SSIE}}$-beta1 possessed far more 
Sn-sites than $(\mathrm{Sn})_{\mathrm{SSIE}}-$ beta2 and $(\mathrm{Sn})_{\mathrm{SSIE}}-$ beta3. The SSIE for $\mathrm{Sn}+\mathrm{Al}$ in deAl-beta1 led to $(\mathrm{SnAl})_{\mathrm{SSIE}}$-beta1 with much lower ratio $\mathrm{Si} / \mathrm{Al}$ and $\mathrm{Sn} / \mathrm{Al}$ than $(\mathrm{Sn})_{\mathrm{SSIE}}$-beta1.

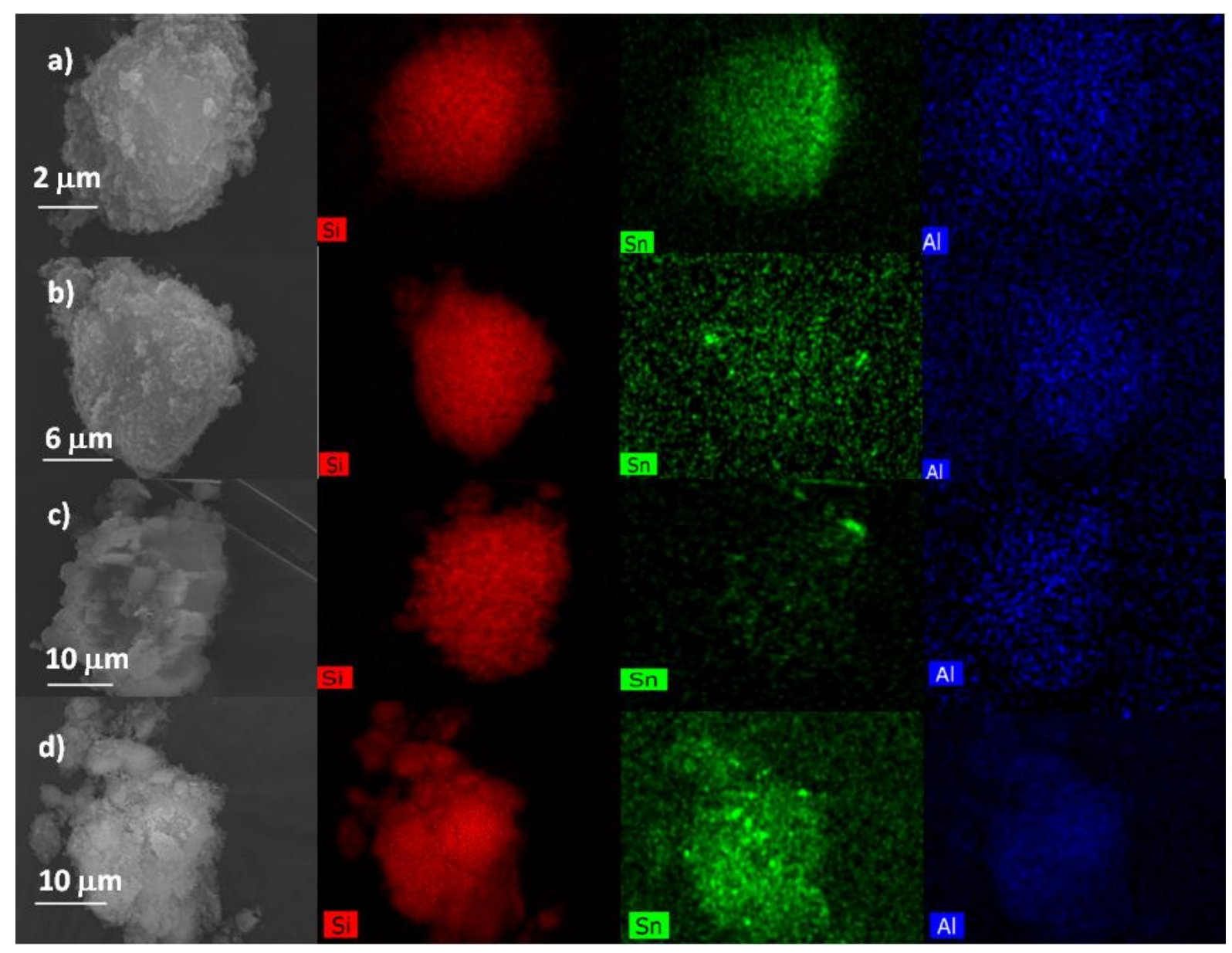

Figure 3. SEM and elemental mapping of $\mathrm{Si}, \mathrm{Al}$ and $\mathrm{Sn}$ for $(\mathrm{Sn})_{\mathrm{SSIE}^{-}}$-beta1 (a), $(\mathrm{Sn})_{\mathrm{SSIE}^{-}}$-beta2

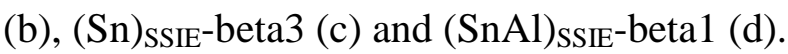

The ${ }^{27} \mathrm{Al}$ MAS NMR spectrum of H-beta exhibits a strong resonance at $53 \mathrm{ppm}$ assigned to framework aluminium species in tetrahedral coordination $\left(\mathrm{Al}_{\text {tetra }}\right)$ and a weak signal at $0 \mathrm{ppm}$ to hexacoordinated $\mathrm{Al}$ species $\left(\mathrm{Al}_{\text {octa }}\right)$ (Figure 4). These results suggest that $\mathrm{H}$-beta possesses essentially Brönsted acidity. The dealumination of $\mathrm{H}$-beta to give deAl-betal leads to a considerable reduction of the amount of $\mathrm{Al}$ species as evidenced by the less resolved spectrum of the latter. For less concentrated acid treatment, the peak assigned to $\mathrm{Al}_{\text {tetra }}$ is well-resolved (exemplified in Figure 4 for deAl-beta2), suggesting that the dealumination was less extensive than for deAl-beta1, and is consistent with the above discussion of ICP- 
AES results (Table 2). The acid-treatment seems to favour the removal of Al species without formation of $\mathrm{Al}_{\text {octa }}$ since the resonance at 0 ppm was always very weak or not observed. The spectral features of deAl-betan and the corresponding materials $(\mathrm{Sn})_{\mathrm{SSIE}}$-betan are similar, suggesting that the $\mathrm{Al}_{\text {tetra }}$ species remaining after dealumination are fairly stable during the

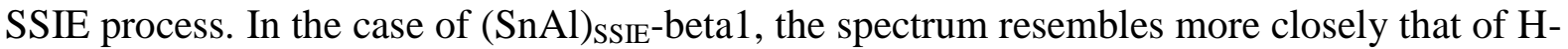
beta than its dealuminated precursor deAl-beta1. These results suggest that reinsertion of $\mathrm{Al}_{\text {tetra }}$ species into the framework of deAl-betal occurred during the SSIE process. On the other hand, the competitive SSIE for tin and aluminium seems to lead to preferential incorporation of $\mathrm{Al}$ into the framework.

Table 2. Elemental analyses and acid properties of the modified beta materials. ${ }^{\text {a }}$

\begin{tabular}{|c|c|c|c|c|c|c|c|c|}
\hline \multirow{3}{*}{ Sample } & \multicolumn{4}{|c|}{ Elemental analyses $^{\mathrm{a}}$} & \multicolumn{4}{|c|}{ Acid properties ${ }^{b}$} \\
\hline & & & & & B & $\mathrm{L}$ & $\mathrm{L}+\mathrm{B}$ & $\mathrm{L} / \mathrm{B}$ \\
\hline & $\mathrm{Si} / \mathrm{Al}$ & $\mathrm{Si} /(\mathrm{Sn}+\mathrm{Al})$ & $\mathrm{Sn} / \mathrm{Al}$ & $\mathrm{Si} / \mathrm{Sn}$ & $\left(\mu \mathrm{mol} . \mathrm{g}^{-1}\right)$ & $\left(\mu \mathrm{mol} . \mathrm{g}^{-1}\right)$ & $\left(\mu \mathrm{mol} . \mathrm{g}^{-1}\right)$ & \\
\hline H-beta & 12.4 & - & - & & $152^{\mathrm{c}}$ & $199^{c}$ & $351^{\mathrm{c}}$ & $1.3^{\mathrm{c}}$ \\
\hline deAl-beta1 & 591 & - & - & & 14 & 2 & 16 & 0.1 \\
\hline deAl-beta2 & 423 & - & - & & 24 & 3 & 27 & 0.1 \\
\hline deAl-beta3 & 222 & - & - & & 38 & 5 & 43 & 0.1 \\
\hline$(\mathrm{Sn})_{\mathrm{SSIE}}$-beta 1 & 553 & 19 & 27.6 & 20 & 7 & 111 & 118 & 15.9 \\
\hline$(\mathrm{Sn})_{\mathrm{SSIE}}$-beta2 & 407 & 154 & 1.6 & 247 & 18 & 12 & 30 & 0.7 \\
\hline$(\mathrm{Sn})_{\mathrm{SSIE}}$-beta3 & 227 & 190 & 0.2 & 1151 & 34 & 7 & 41 & 0.2 \\
\hline$(\mathrm{SnAl})_{\mathrm{SSIE}}$-beta 1 & 30 & 22 & 0.4 & 81 & 113 & 133 & 246 & 1.2 \\
\hline
\end{tabular}

\footnotetext{
${ }^{a}$ Molar ratios determined by ICP-AES. ${ }^{b}$ Determined by FT-IR of adsorbed pyridine, at 150

${ }^{\circ} \mathrm{C} ; \mathrm{B}=$ Brönsted acid sites, $\mathrm{L}=$ Lewis acid sites, $\mathrm{B}+\mathrm{L}=$ total amount of acid sites. ${ }^{\mathrm{c}}$ Results reported in ref. 41.
} 


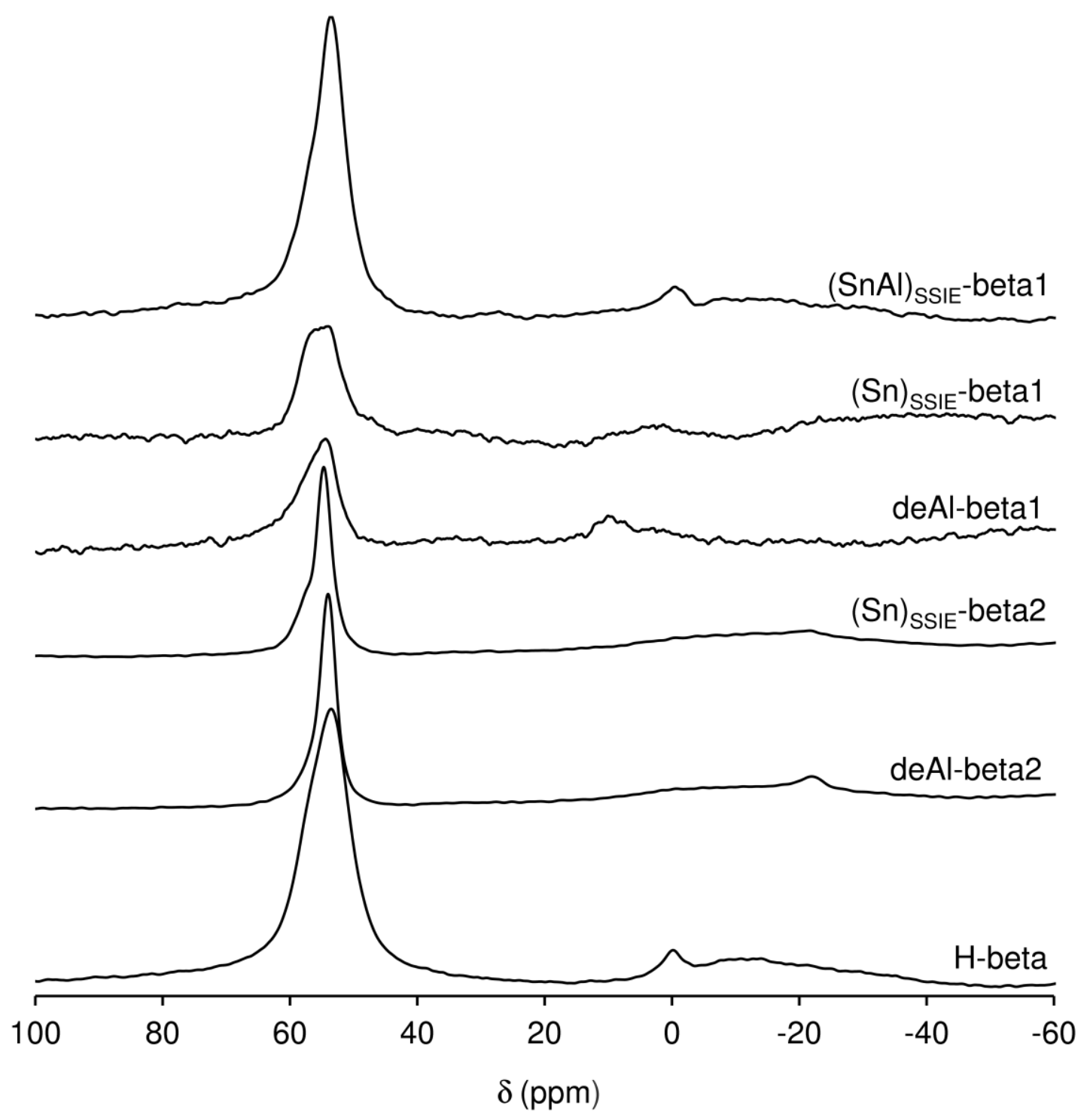

Figure 4. ${ }^{27} \mathrm{Al}$ MAS NMR spectra of H-beta and its modified versions.

The FT-IR spectra of the beta materials exhibited characteristic vibrational modes associated with the zeolite structure [94-97]. A comparison of the FT-IR spectra of deAl-betan and the corresponding Sn-containing materials shows decreased relative intensity of the band at ca. $950 \mathrm{~cm}^{-1}$ after SSIE (Figure 5). This band can be assigned to $\mathrm{Si}-\mathrm{O}$ stretching vibrations of $\mathrm{Si}-\mathrm{OH}$ groups present at connectivity defects [98]. Accordingly, the SSIE process resulted in the grafting of Sn and/or Al at defect sites of the dealuminated solids. In general, after SSIE a band at ca. $575 \mathrm{~cm}^{-1}$ appeared with enhanced relative intensity, which may be related to enhanced framework vibrations after the SSIE process [97, 98]. Bulk $\mathrm{SnO}_{2}$ exhibits a very 
broad band below $750 \mathrm{~cm}^{-1}$ (roughly centred at ca. $620 \mathrm{~cm}^{-1}$ ). In this spectral region, all modified beta materials (Sn-free and Sn-containing) exhibit weak bands, making it difficult to make unambiguous assignments.

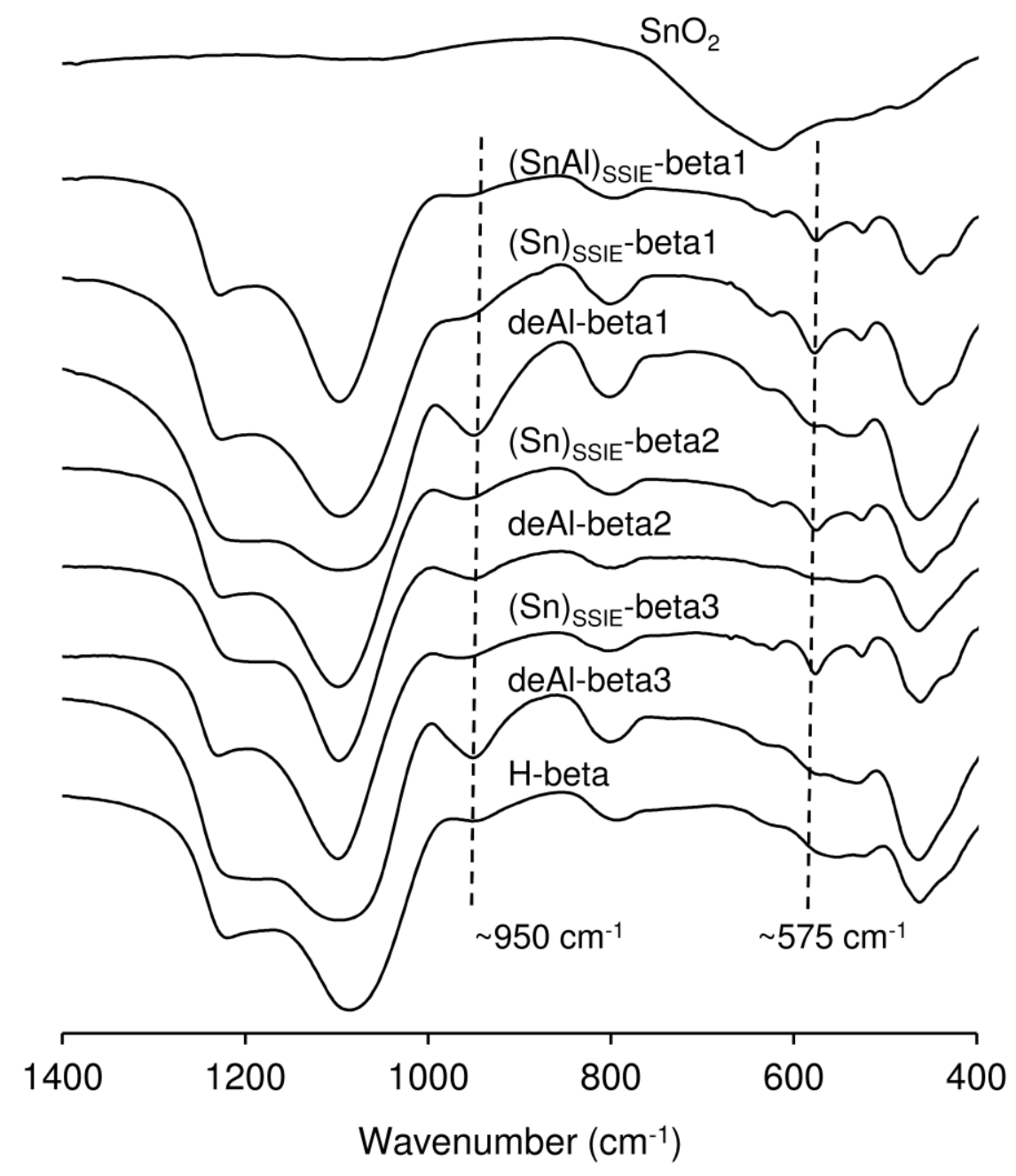

Figure 5. FT-IR ATR spectra of $\mathrm{H}$-beta and its modified versions, and $\mathrm{SnO}_{2}$ for comparison.

The diffuse reflectance UV-vis spectra of the beta materials and, for comparison, that of $\mathrm{SnO}_{2}$ are given in Figure 6 . The material $(\mathrm{Sn})_{\mathrm{SSIE}}$-beta1 exhibits a prominent broad band centred at $256 \mathrm{~nm}$, which can be attributed to charge transfer transitions from $\mathrm{O}^{2-}$ to $\mathrm{Sn}^{4+}$ of isolated tetrahedral $\mathrm{Sn}^{4+}$ species [99]. This band was hardly detected for the Sn-free samples, namely, deAl-beta1 and H-beta. The materials $(\mathrm{Sn})_{\mathrm{SSIE}^{-}}$-beta2, $(\mathrm{Sn})_{\mathrm{SSIE}}$-beta3 and $(\mathrm{SnAl})_{\mathrm{SSIE}^{-} \text {-beta1 }}$ exhibited bands characteristic of bulk $\mathrm{SnO}_{2}$ which are poorly detected in the spectrum of $(\mathrm{Sn})_{\mathrm{SSIE}}$-beta1. 


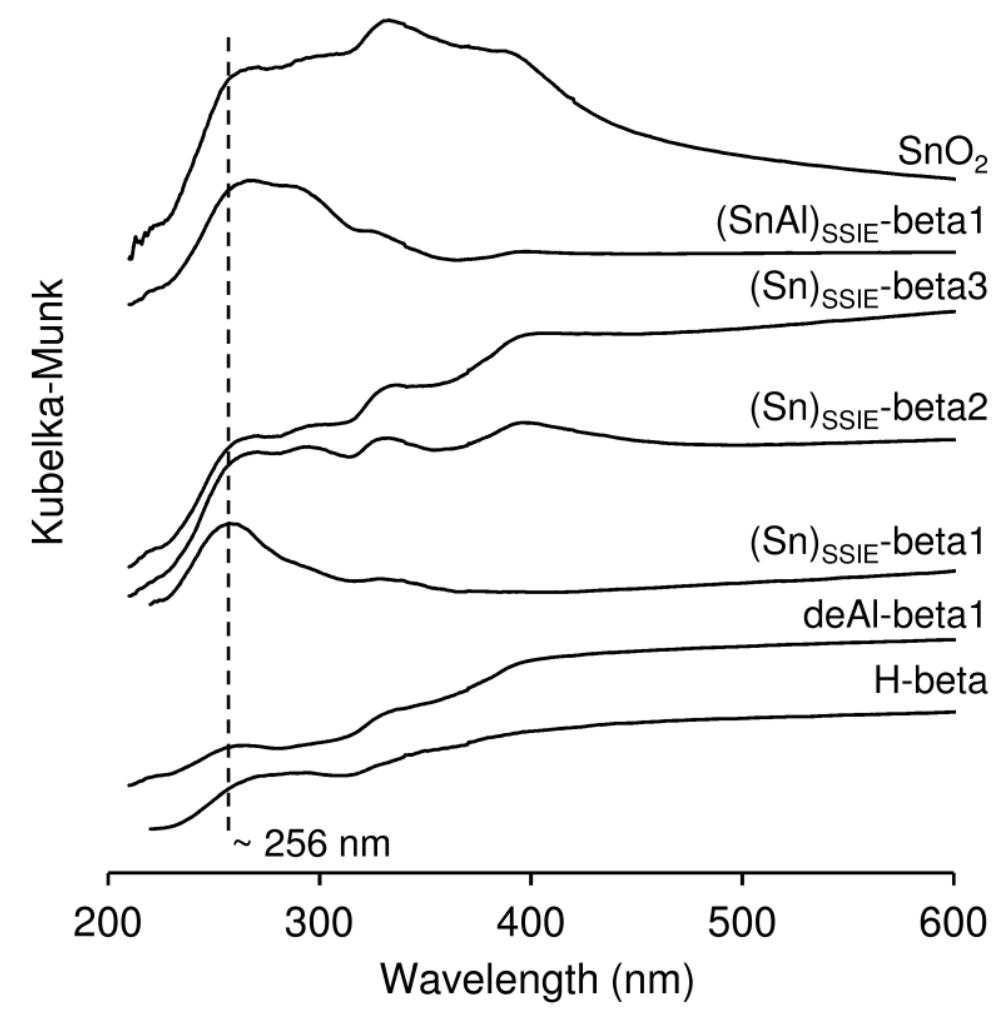

Figure 6. Diffuse reflectance UV-vis spectra of $\mathrm{H}$-beta and its modified versions, and $\mathrm{SnO}_{2}$ for comparison.

Figure 7 shows the UV-Raman spectra of the beta materials and bulk $\mathrm{SnO}_{2}$. The bands characteristic of the BEA framework structure at $316-330 \mathrm{~cm}^{-1}, 340-345 \mathrm{~cm}^{-1}, 402-405 \mathrm{~cm}^{-1}$, 426-436 $\mathrm{cm}^{-1}, 470-475 \mathrm{~cm}^{-1}$ and $818-826 \mathrm{~cm}^{-1}[100,101]$ remain present after the dealumination and SSIE. These results are consistent with the powder XRD data in that the BEA framework structure was preserved during the modification treatments. The spectrum of bulk $\mathrm{SnO}_{2}$ displays two strong bands at 470 and $622 \mathrm{~cm}^{-1}$. According to the literature, nanocrystalline cassiterite $\mathrm{SnO}_{2}$ (particle sizes of 10-15 nm) exhibits a band in the range 634$641 \mathrm{~cm}^{-1}$ which blue-shifts due to decreasing particle sizes [102]. The Sn-free beta samples exhibit a band at ca. $470 \mathrm{~cm}^{-1}$, and no band appears in the range $600-650 \mathrm{~cm}^{-1}$. Thus, the latter spectral range can be used to trace crystalline $\mathrm{SnO}_{2}$ in the SSIE beta samples. On the other hand, framework Sn-sites can give rise to a Raman band at ca. $705 \mathrm{~cm}^{-1}$ [82], which appears for all Sn-containing beta samples; however, unambiguous assignment of this band is 
difficult since a band at similar wavenumbers appears in the spectrum of deAl-beta1. In contrast to $(\mathrm{Sn})_{\mathrm{SSIE}}$-beta1, the materials $(\mathrm{Sn})_{\mathrm{SSIE}}-$ beta2 and $(\mathrm{Sn})_{\mathrm{SSIE}}$-beta3 exhibited a more prominent band at ca. $640 \mathrm{~cm}^{-1}$, likely due to the formation of $\mathrm{SnO}_{2}$ nanoparticles. The spectrum of $(\mathrm{SnAl})_{\mathrm{SSIE}^{-}}$betal shows very broad bands in the range $600-780 \mathrm{~cm}^{-1}$ (centred at ca. $635 \mathrm{~cm}^{-1}$ and $749 \mathrm{~cm}^{-1}$ ), which may be due to tin-containing particles [82, 102]. The UVRaman data are consistent with the results obtained by powder XRD, EDS mapping and UVVis optical response: the dispersion of $\mathrm{Sn}$ seems more homogeneous for $(\mathrm{Sn})_{\mathrm{SSIE}^{-}}$-beta1 than the remaining Sn-containing materials.

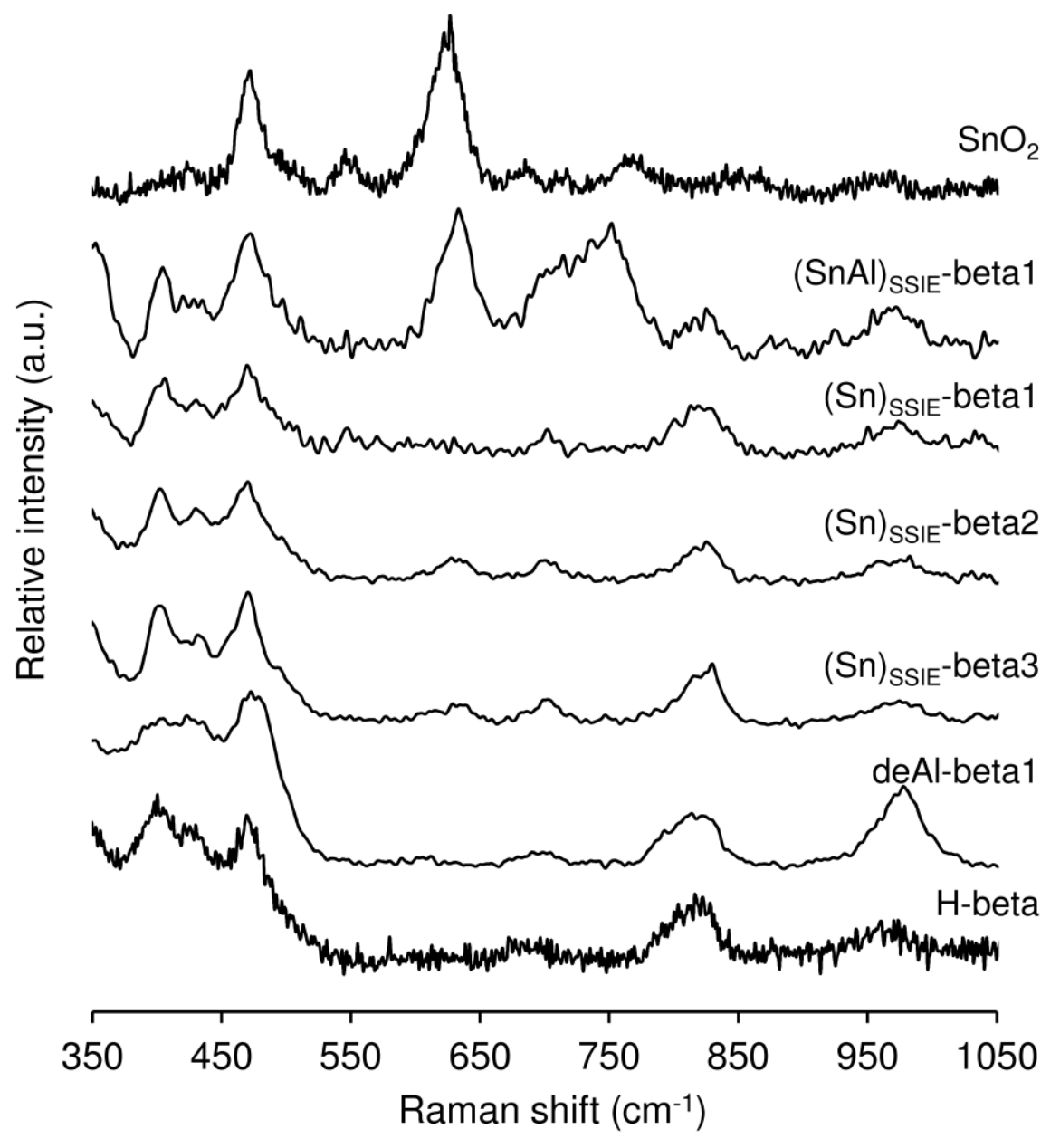

Figure 7. UV-Raman spectra of $\mathrm{H}$-beta and its modified versions, and $\mathrm{SnO}_{2}$ for comparison.

For insights into the chemical environment of the Sn-sites of the modified beta materials, XPS measurements were carried out also on bulk $\mathrm{SnO}_{2}$ for comparison. 
The XPS chemical analyses of the Sn-containing beta materials indicated increasing $\mathrm{Si} / \mathrm{Sn}$ surface atomic ratio in the order $(\mathrm{Sn})_{\mathrm{SSIE}}$-beta1 $(23)<(\mathrm{SnAl})_{\mathrm{SSIE}}$-beta1 $(57)<(\mathrm{Sn})_{\mathrm{SSIE}}$-beta2 $(178)<(\mathrm{Sn})_{\mathrm{SSIE}}-$ beta3 $(358)$. This trend is the same as that for $\mathrm{Si} / \mathrm{Sn}$ determined by ICP-AES. The values of the ratio $\mathrm{Si} / \mathrm{Sn}$ determined by ICP-AES and XPS were similar for $(\mathrm{Sn})_{\mathrm{SSIE}^{-}}$ beta1, indicating homogeneous dispersion of $\mathrm{Sn}$ in this material. Conversely, for $(\mathrm{Sn})_{\mathrm{SSIE}^{-}}$ beta2, $(\mathrm{Sn})_{\mathrm{SSIE}^{-}}$-beta3 and $(\mathrm{SnAl})_{\mathrm{SSIE}^{-}}$-beta1, XPS indicated lower Si/Sn than ICP-AES, and thus the dispersion of $\mathrm{Sn}$ in these materials was heterogeneous. These differences of $\mathrm{Sn}$ dispersion are consistent with the above powder XRD, EDS mapping, UV-Vis and UVRaman studies of the prepared materials.
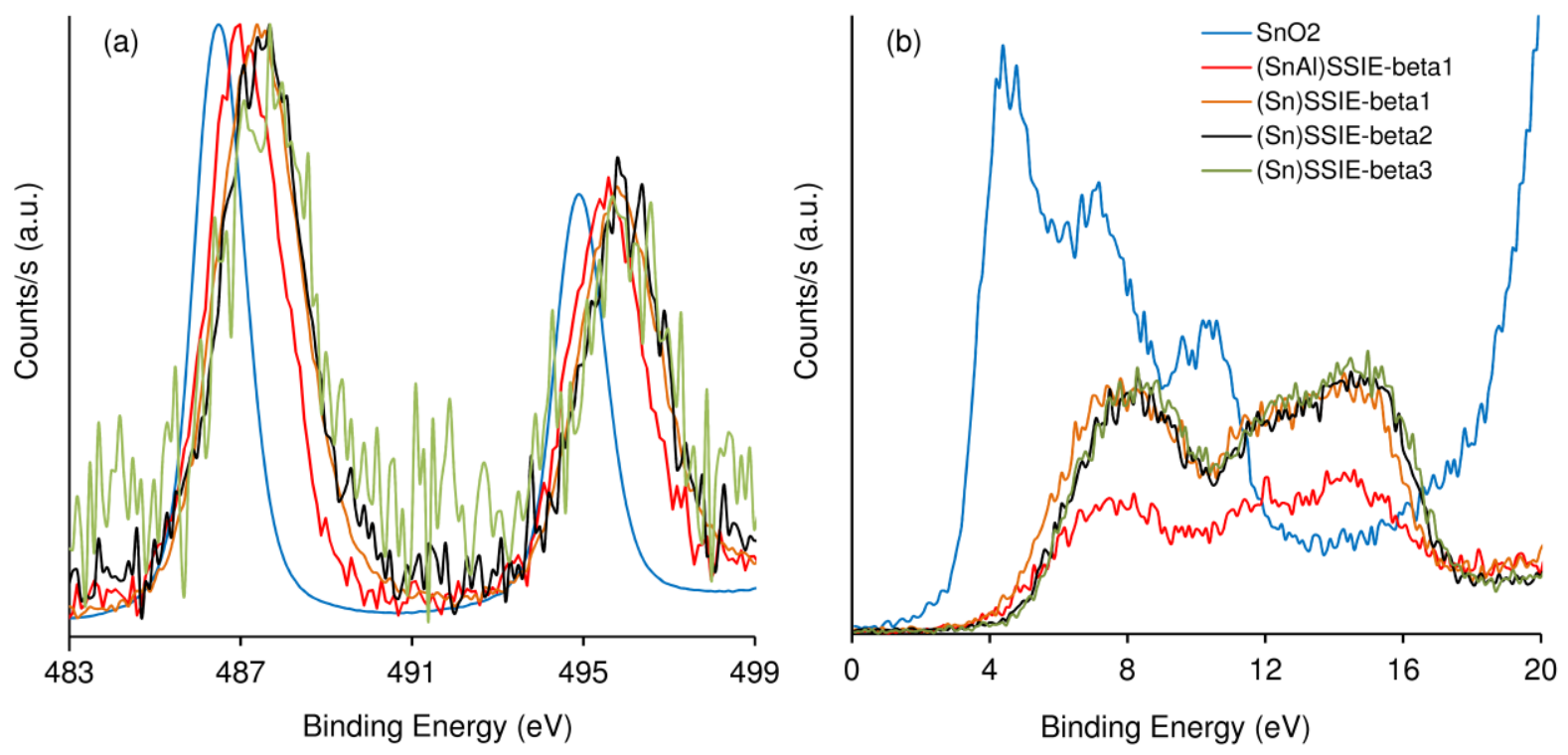

Figure 8. Sn 3d XPS core level (a) and valence (b) spectra for bulk $\mathrm{SnO}_{2}$ and Sn-containing beta materials. The legend applies for (a) and (b).

The XPS core level spectra of the Sn-containing samples are shown in Figure 8 (a). The $(\mathrm{Sn})_{\operatorname{SSIE}}$-betan materials exhibited two signals referent to the $\mathrm{Sn} 3 \mathrm{~d}_{5 / 2}$ and $\mathrm{Sn} 3 \mathrm{~d}_{3 / 2}$ photoelectrons, centered at higher binding energy values (ca. $487.5 \mathrm{eV}$ and ca. $495.8 \mathrm{eV}$ ) than bulk $\mathrm{SnO}_{2}$, located at $486.5 \mathrm{eV}$ and ca. $495 \mathrm{eV}$. Based on literature data, these spectral features suggest the successful incorporation of tetrahedral Sn sites into the zeolitic 
framework [103-105]. The FWHM of the Sn 3d 5/2 shifted band of the modified beta materials is significant (wider than $2.0 \mathrm{eV}$ ), which may be due to moderate chemical/configurational disorder of the Sn-sites. The material $(\mathrm{SnAl})_{\mathrm{SSIE}}$-betal exhibited a relatively broad bands centered at lower binding energies (at ca. 486.8 and $495.5 \mathrm{eV}$ ) in relation to the remaining materials. The XPS valence spectra of all the modified beta materials are similar to each other, but different from bulk $\mathrm{SnO}_{2}$ (Figure 8 (b)) [103]. Noteworthy, distinction between $\operatorname{Sn}(\mathrm{IV})$ and lower valency tin species is not possible by XPS [105]. Overall, the XPS results together with the above characterization studies indicate that different amounts of tin were incorporated into the frameworks of the dealuminated materials deAl-betan.

The acid properties of the modified beta materials were measured by FT-IR of pyridine adsorbed at $150{ }^{\circ} \mathrm{C}$ (Table 2). The dealumination of H-beta led to considerable reduction of total amount of $(\mathrm{L}+\mathrm{B})$ acid sites, which followed the order deAl-beta $1<$ deAl-beta $<$ deAlbeta3, which is similar to that of the inversed molar ratios $\mathrm{Si} / \mathrm{Al}$. The materials deAl-betan possessed low Brönsted acidity and negligible Lewis acidity, consistent with the ${ }^{27} \mathrm{Al}$ MAS NMR data (Figure 4) which showed predominance of tetrahedral Al-sites (which possess Brönsted acid character).

The amounts of B acid sites of deAl-betan were similar before and after SSIE for Sn, suggesting that the B acid sites were fairly well preserved during the SSIE procedure. On the other hand, the amount of L acid sites increased drastically for $(\mathrm{Sn})_{\mathrm{SSIE}}$-beta1 (L/B=15.9), and thus this material was rich in Lewis acid $\mathrm{Sn}$-sites. For each of the remaining $(\mathrm{Sn})_{\mathrm{SSIE}}$-betan materials, the total amount of $(\mathrm{L}+\mathrm{B})$ acid sites was roughly comparable to that of the corresponding deAl-betan, and possessed slightly higher ratio L/B. These results correlated with the ICP-AES data, which indicated low amounts of Al-and $\mathrm{Sn}$-sites (high $\mathrm{Si}(\mathrm{Sn}+\mathrm{Al})$ ratios) in $(\mathrm{Sn})_{\mathrm{SSIE}}$-beta2 and $(\mathrm{Sn})_{\mathrm{SSIE}^{-}}$-beta3. 
The SSIE for $(\mathrm{Sn}+\mathrm{Al})$ gave $(\mathrm{SnAl})_{\mathrm{SSIE}}$-beta1 with much higher total amount of $(\mathrm{L}+\mathrm{B})$ acid sites than $(\mathrm{Sn})_{\mathrm{SSIE}}$-beta1, mainly due to enhanced Brönsted acidity (incorporated Al-sites) (Table 2). On the other hand, the high amount of $\mathrm{L}$ acid sites and very low $\mathrm{Sn} / \mathrm{Al}$ ratio of $(\mathrm{SnAl})_{\mathrm{SSIE}}$-beta1 (e.g. in comparison to $(\mathrm{Sn})_{\mathrm{SSIE}}$-beta1), suggests that the Lewis acidity of $(\mathrm{SnAl})_{\mathrm{SSIE}}$-beta1 was mainly due to $\mathrm{Al}$-sites. The materials $(\mathrm{SnAl})_{\mathrm{SSIE}}$-beta1 $(\mathrm{Si} / \mathrm{Al}=30)$ and $\mathrm{H}$-beta $(\mathrm{Si} / \mathrm{Al}=12.4)$ were more similar in terms of $\mathrm{Si} / \mathrm{Al}$ and $\mathrm{L} / \mathrm{B}$ ratios, and exhibited comparable ${ }^{27} \mathrm{Al}$ MAS NMR spectra (Figure 4), supporting the predominance of acid Al-sites in $(\mathrm{SnAl})_{\mathrm{SSIE}}$-beta1.

\subsection{Catalytic studies}

\subsubsection{General considerations}

The modified beta materials were tested as catalysts in the one-pot conversion of Fur to bioproducts under a consistent reaction condition $\left(120{ }^{\circ} \mathrm{C}, 0.45 \mathrm{M}\right.$ substrate in 2-butanol $\left.(2 \mathrm{BuOH}), 26.7 \mathrm{~g}_{\text {cat }} \mathrm{L}^{-1}\right)$. The conversion of Fur in the alcohol media gives FA, FEs, LEs, LA, AnLs and GVL (Scheme 1). Of the prepared (Sn,Al)-containing beta materials, $(\mathrm{Sn})_{\mathrm{SSIE}}$-beta1 was the best-performing catalyst in the one-pot conversion of Fur, leading to faster Fur conversion and high yields of bio-products $(83 \%$ bio-products yield at $86 \%$ conversion, Figure 9). The main bio-product was $2 \mathrm{BMF}$ formed with up to $58 \%$ yield ( $5 \mathrm{~h}$ reaction); additionally, AnLs, 2BL, LA and GVL were formed with increasing yields (23, 11, 14 and $2 \%$, respectively, at $24 \mathrm{~h}$ ). The isomerisation of $\alpha \mathrm{AnL}$ to $\beta \mathrm{AnL}$ occurred with time, which is consistent with the greater stability of the latter due to conjugation of its $\mathrm{C}-\mathrm{C}$ double bond with the C-O double bond [106]. 


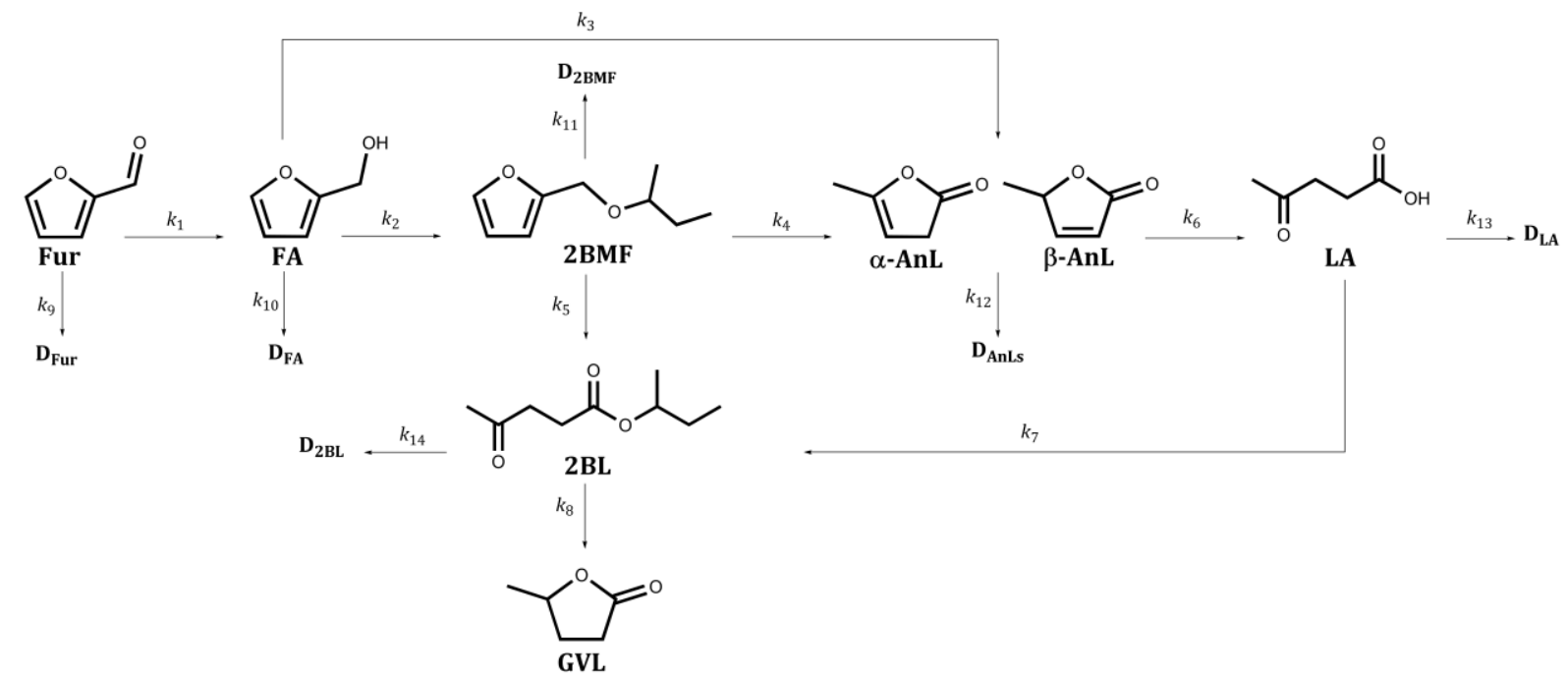

Scheme 1. Conversion of hemicelluloses-derived furfural (Fur) in alcohol media to give useful bio-products via acid and reduction chemical routes.

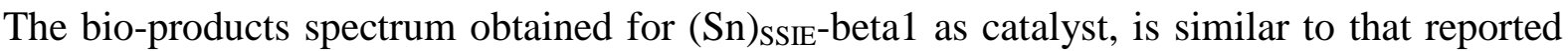
by Román-Leshkov et al. [4] for the same reaction (Fur/2-BuOH, at $120^{\circ} \mathrm{C}$ ), using a mixture of different catalysts, namely (Al-free) Zr-beta (prepared via 40 day hydrothermal synthesis) and Al-MFI (the two materials were used in a mass ratio of 1:2). The Zr-beta material was important for the reduction steps via catalytic transfer hydrogenation $(\mathrm{CTH})$ with the $\mathrm{H}$-donor solvent, and, on the other hand, Al-MFI promoted acid-catalysed steps [4]. Although the spectrum of bio-products was the same for the two catalytic systems, significant differences in reaction kinetics and bio-products yields were observed. The mixed catalysts led mainly to LA formation in an initial stage, and after $5 \mathrm{~h}$ reaction the predominant bio-product was GVL (68\% yield at $24 \mathrm{~h}$ ) [4]. Conversely, $(\mathrm{Sn})_{\mathrm{SSIE}}$-beta1 led to very low GVL yields throughout 24 $\mathrm{h}$ reaction, and the main bio-product was $2 \mathrm{BMF}$. The $(\mathrm{Sn})_{\mathrm{SSIE}}$-betal catalyst was used in approximately a quarter of the total mass of mixed catalysts reported in ref. [4], which can impact on the overall kinetics. On the other hand, the catalytic materials' properties are different, which can affect the bio-products distributions. For example, the catalysts possessed different transition metal (M) sites $(\mathrm{M}=\mathrm{Sn}$ or $\mathrm{Zr})$, and the intrinsic activities of $\mathrm{Zr}$ - 
or Sn-sites for a specific step may be different; the $\mathrm{Si} / \mathrm{M}$ atomic ratio was greater for $\mathrm{Zr}$-beta

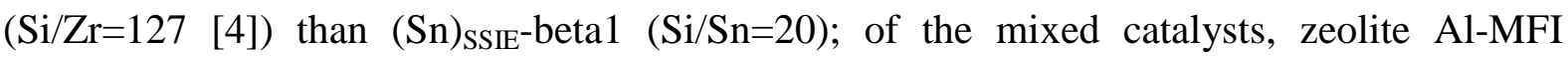
possessed much higher $\mathrm{Si} / \mathrm{Al}$ atomic ratio $(\mathrm{Si} / \mathrm{Al}=17[4])$ than $(\mathrm{Sn})_{\mathrm{SSIE}}$-beta1 $(\mathrm{Si} / \mathrm{Al}=553)$, and was used in more than double the mass amount of $(\mathrm{Sn})_{\text {SSIE}}-$ betal used in the present work, which can impact on the acid-catalysed pathways of the overall process. Moreover, reactions of the reagents/intermediates over a sole solid material, or two different materials in the reaction medium, may involve different competitive and/or cooperative effects. In order to help understand the influence of the catalytic properties on specific reaction pathways in the overall reaction system, a systematic catalytic study has been carried out for the prepared materials with different bio-products as substrates, and discussed in section 3.2.2.
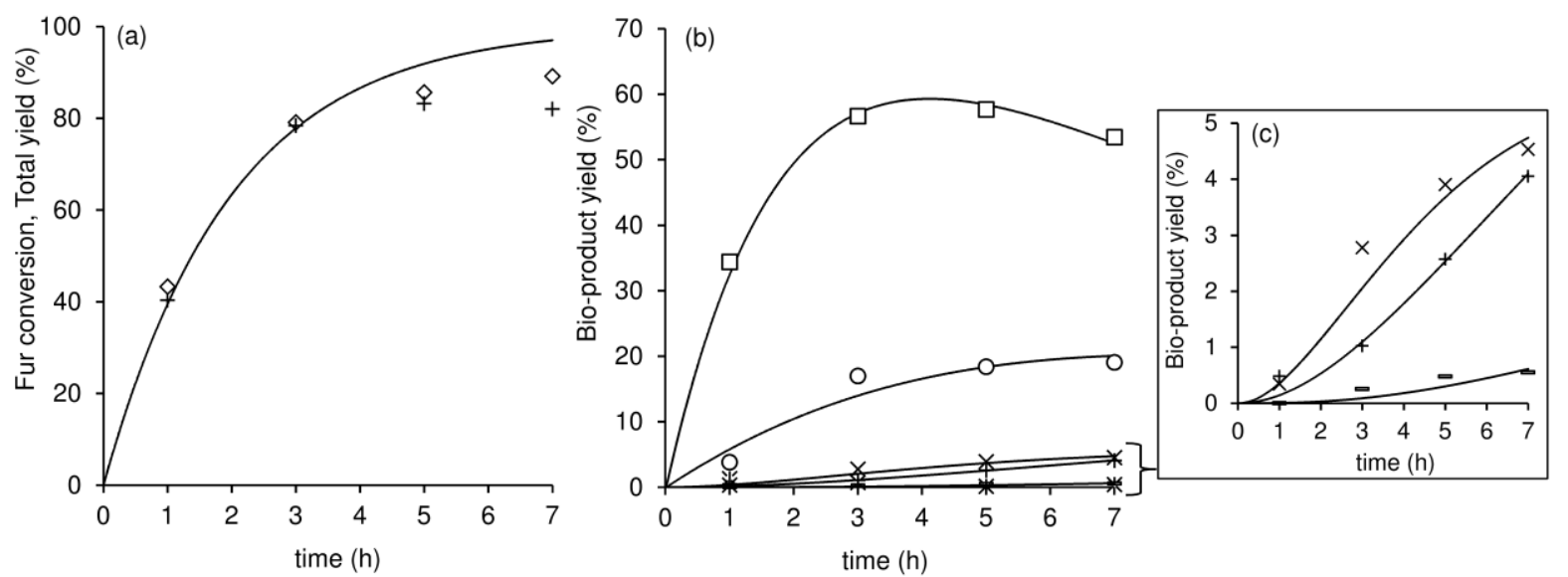

Figure 9. One-pot conversion of Fur in 2-butanol medium using $(\mathrm{Sn})_{\mathrm{SSIE}}$-beta1 as catalyst: (a) dependency of Fur conversion $(\diamond)$ and total yield of bio-products $(+)$ on reaction time; (b) dependency of the yield of FA (*), 2BMF $(\square), \beta A n L(o), \alpha A n L(\diamond)$, LA (+), 2BL $(\times)$ and GVL (-) on reaction time, with (c) showing an expanded plot for LA (+), 2BL (x) and GVL (). The markers are experimental data, and the calculated kinetic curves are given for Fur conversion (a), and bio-products yields ((b) and (c)). Reaction conditions: $[\mathrm{S}]_{0}=0.45 \mathrm{M}$ in $2 \mathrm{BuOH}$, catalyst load $=27 \mathrm{~g}_{\text {cat }} \mathrm{L}^{-1}, 120^{\circ} \mathrm{C}$. 
The catalytic stability is an important factor which can affect the overall reaction process. The stability of $(\mathrm{Sn})_{\mathrm{SSIE}}$-betal was investigated for the reaction of Fur in $2 \mathrm{BuOH}$, at $120^{\circ} \mathrm{C}$. From the kinetic profiles it is noticeable that the catalytic reaction slowed down after ca. $5 \mathrm{~h}$ (86\% conversion), and the total yield of bio-products remained roughly constant (conversion was $86-95 \%$ between 5 and $24 \mathrm{~h}$, and the total yield was $81-83 \%$, Figure 9). These results may be partly due to partial catalyst deactivation by adsorbed carbonaceous matter (byproducts) since the originally white powdered catalyst turned brownish in colour during the catalytic reaction. Thermal analyses (DSC, TGA, Figure S3) of the used catalyst ( $24 \mathrm{~h}$ batch run) indicated exothermic processes occurring above ca. $350{ }^{\circ} \mathrm{C}$, which were not observed for the original catalyst, and may be attributed to the decomposition of organic matter. On the other hand, a mass loss of ca. $5 \mathrm{wt} . \%$ in the temperature range $350-650{ }^{\circ} \mathrm{C}$ (determined by TGA) was observed for the used catalyst, whereas no mass loss was observed for the original catalyst in the same temperature interval. The observed mass loss was similar to the mass loss of Fur converted to by-products (at $24 \mathrm{~h}$ reaction), and thus reaction by-products were mostly deposited on the solid catalyst. A thermal treatment was applied to the used catalyst, which allowed effective catalyst regeneration, i.e., steady catalytic activity for six consecutive batch runs, without changes in the distribution of the bio-products (Figure 10). ICP-AES analyses showed that the original and used catalysts had similar Si/Al molar ratios (553 and 566 for $(\mathrm{Sn})_{\mathrm{SSIE}^{-}}$-beta1 and $(\mathrm{Sn})_{\mathrm{SSIE}}$-beta1(used), respectively), and no reduction of tin content. A 24 h-contact test for $(\mathrm{Sn})_{S S I E}$-beta1 (details given in the experimental section) revealed no catalytic contribution from the liquid phase, indicating that the catalytic reaction is truly heterogeneous. 


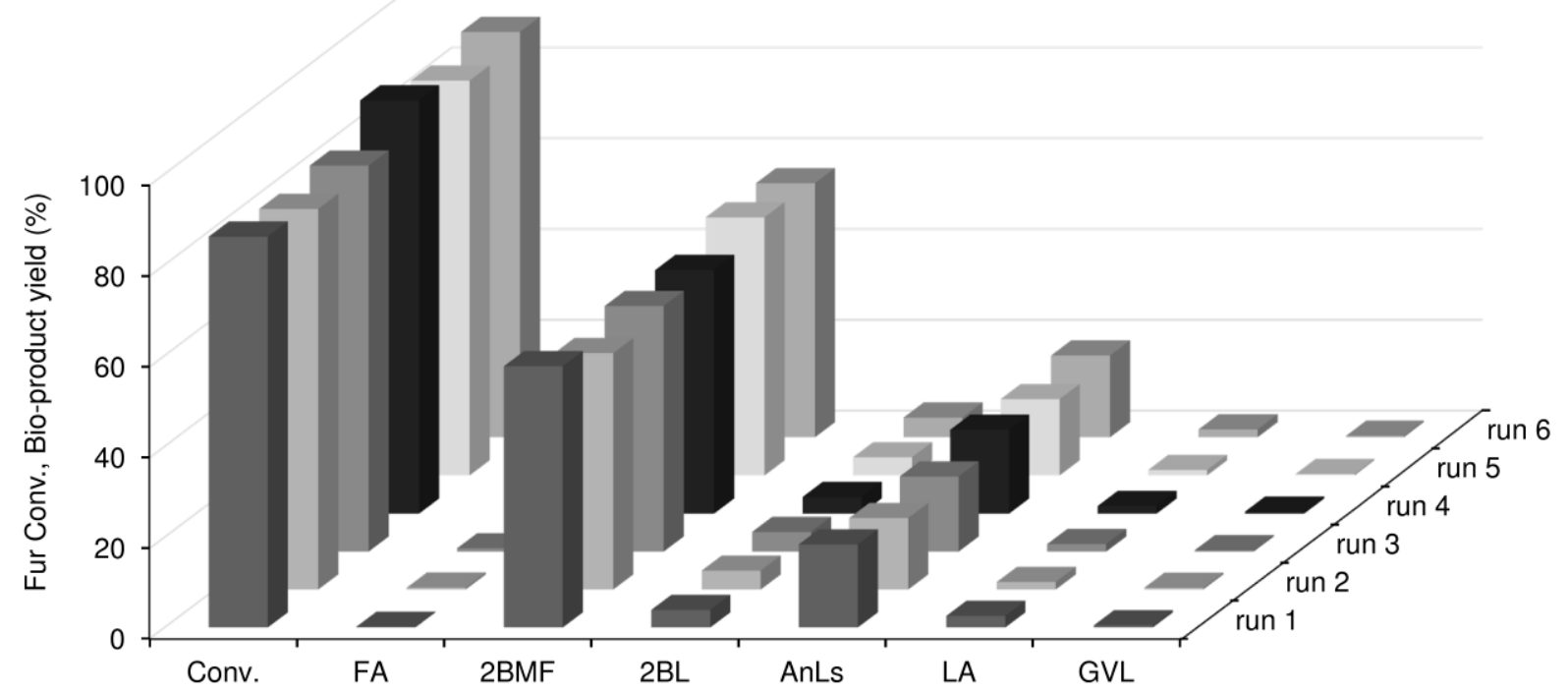

Figure 10. Catalytic performance of $(\mathrm{Sn})_{\mathrm{SSIE}}$-betal in the one-pot conversion of Fur in 2butanol for six consecutive 5 h-batch runs. Reaction conditions: $[\mathrm{S}]_{0}=0.45 \mathrm{M}$ in $2 \mathrm{BuOH}$, catalyst load $=27 \mathrm{~g}_{\text {cat }} \mathrm{L}^{-1}, 120^{\circ} \mathrm{C}$.

The type of solvent is an important parameter of the process. The formation of FA from Fur [107-109], and the conversion of LEs to GVL involve reduction chemistry $(\mathrm{CTH})[72,110]$, where the solvent can simultaneously act as $\mathrm{H}$-donor reagent $[111,112] .2 \mathrm{BuOH}$ and 2propanol $(2 \mathrm{PrOH})$ are considered favourable H-donor solvents, which may avoid side reactions $[110,111]$. Hence, a comparative study was carried out for $2 \mathrm{BuOH}$ and $2 \mathrm{PrOH}$ as reacting solvents for the one-pot conversion of Fur in the presence of $(\mathrm{Sn})_{\mathrm{SSIE}}$-beta1 (Table $\mathrm{S} 1)$. The catalytic reaction in $2 \mathrm{BuOH}$ was faster and led to higher total yield of bio-products than that in $2 \mathrm{PrOH}(36 \%$ yield at $79 \%$ Fur conversion, $24 \mathrm{~h}$ ). A similar trend in terms of reaction rate was reported in the literature for the conversion of methyl levulinate to GVL, in the presence of $\mathrm{Zr}$-beta, in $2 \mathrm{PrOH}$ or $2 \mathrm{BuOH}$, at $120^{\circ} \mathrm{C}$ [4]. The solvent effect was also investigated for the reactions starting from FA and LEs, in the presence of $(\mathrm{Sn})_{\mathrm{SSIE}^{-}}$beta1, at $120{ }^{\circ} \mathrm{C}$. With FA as substrate and $2 \mathrm{BuOH}$ or $2 \mathrm{PrOH}$ as reacting solvent, the catalytic results were similar (Table S1). These results somewhat parallels the literature data for the one-pot 
conversion of $\mathrm{HMF}$ in the presence of (Al-free) Sn-beta, in $2 \mathrm{PrOH}$ versus $2 \mathrm{BuOH}$ medium [113]. With LEs as substrates, $(\mathrm{Sn})_{\mathrm{SSIE}}$-beta1 led to faster reaction in $2 \mathrm{PrOH}$ than in $2 \mathrm{BuOH}$, and similar GVL yields were reached at $24 \mathrm{~h}$ reaction (e.g. with $1 \mathrm{BL}$ as substrate, the GVL yields were in the range 55-59\%). Based on the different trends, the influence of the reacting solvent on multistep conversion processes is not straightforward, and may be due to interplay of different factors, including the reactivities of reagents and intermediates under the reaction conditions, and catalytic material properties. Further catalytic studies of the prepared materials were carried out using $2 \mathrm{BuOH}$ as $\mathrm{H}$-donor.

\subsubsection{Influence of material properties on the catalytic pathways}

In order to help understand the effects of different properties of the modified beta catalysts on specific reaction pathways in the overall reaction system, the prepared materials were tested as catalysts for the reactions starting from FA, FEs (EMF and 1BMF), LEs (1BL and EL), $\alpha A n L$ and LA. For all substrates tested, the reactions without catalyst led to negligible or very low yields of bio-products at $24 \mathrm{~h}$ (Table 3). Similar poor catalytic results were observed for all substrates using (bulk) $\mathrm{SnO}_{2}$ as catalyst, suggesting that octahedral Sn-sites are not effective for the various reduction and acid steps involved in the overall process.

Table 3. Catalytic performance of zeolite H-beta and related modified versions as well as $\mathrm{SnO}_{2}$ in the reaction of Fur and selected bio-products as substrates (S), at $120^{\circ} \mathrm{C} .{ }^{\mathrm{a}}$

\begin{tabular}{|c|c|c|c|c|c|c|c|c|c|}
\hline \multirow{2}{*}{ Sample } & \multirow{2}{*}{$\mathrm{S}$} & \multirow{2}{*}{$\begin{array}{c}\text { Conv. }^{\mathrm{b}} \\
(\%)\end{array}$} & \multicolumn{6}{|c|}{ Bio-products yield (\%) } & \multirow{2}{*}{$\begin{array}{l}\text { Total } \\
\text { yield } \\
(\%)^{\mathrm{c}}\end{array}$} \\
\hline & & & FA & 2BMF & $2 \mathrm{BL}$ & LA & AnLs & GVL & \\
\hline$(\mathrm{Sn})_{\mathrm{SSIE}}$-beta1 & Fur & $(86)^{\mathrm{d}} 95$ & $(<1)^{\mathrm{d}} 1$ & $(58)^{\mathrm{d}} 29$ & $(4)^{\mathrm{d}} 11$ & $(3)^{\mathrm{d}} 14$ & $(18)^{\mathrm{d}} 23$ & $(<1)^{\mathrm{d}} 2$ & $(83)^{\mathrm{d}} 80$ \\
\hline$(\mathrm{Sn})_{\mathrm{SSIE}}{ }^{-b e t a} 2$ & Fur & 36 & 1 & 1 & 5 & 2 & 8 & - & 17 \\
\hline$(\mathrm{Sn})_{\mathrm{SSIE}}-\mathrm{beta} 3$ & Fur & 32 & 1 & - & 2 & - & 7 & - & 10 \\
\hline$(\mathrm{SnAl})_{\mathrm{SSIE}}$-beta1 & Fur & 26 & 1 & - & 4 & 2 & 9 & - & 16 \\
\hline deAl-beta1 & Fur & 24 & 1 & 2 & 1 & - & 1 & - & 5 \\
\hline
\end{tabular}




\begin{tabular}{|c|c|c|c|c|c|c|c|c|c|}
\hline deAl-beta2 & Fur & 22 & 1 & $<1$ & 1 & - & $<1$ & - & 2 \\
\hline deAl-beta3 & Fur & 22 & 1 & $<1$ & 1 & - & 1 & - & 3 \\
\hline H-beta & Fur & 43 & 1 & - & 3 & 2 & 4 & - & 10 \\
\hline $\mathrm{SnO}_{2}$ & Fur & 4 & - & - & - & - & - & - & - \\
\hline none & Fur & 9 & 1 & - & - & - & - & - & 1 \\
\hline$(\mathrm{Sn})_{\mathrm{SSIE}^{-}}-\mathrm{beta} 1$ & FA & $(100)^{\mathrm{e}} 100$ & - & $(44)^{\mathrm{e}} 20$ & $(2)^{\mathrm{e}} 6$ & $(1)^{\mathrm{e}} 6$ & $(16)^{\mathrm{e}} 17$ & $(-)^{\mathrm{e}} 1$ & $(63)^{\mathrm{e}} 50$ \\
\hline$(\mathrm{Sn})_{\mathrm{SSIE}}-\mathrm{beta} 2$ & FA & 100 & - & 19 & 8 & 2 & 16 & - & 45 \\
\hline$(\mathrm{Sn})_{\mathrm{SSIE}}-\mathrm{beta} 3$ & FA & 100 & - & 14 & 9 & 3 & 16 & - & 42 \\
\hline$(\mathrm{SnAl})_{\mathrm{SSIE}}$-beta1 & FA & 100 & - & 1 & 8 & 5 & 22 & - & 36 \\
\hline deAl-beta1 & FA & 97 & - & 47 & 6 & - & 7 & - & 60 \\
\hline deAl-beta2 & FA & 100 & - & 24 & 14 & 3 & 16 & - & 57 \\
\hline deAl-beta3 & FA & 100 & - & 32 & 8 & 4 & 11 & - & 55 \\
\hline H-beta & FA & 100 & - & - & 13 & 14 & 15 & - & 42 \\
\hline $\mathrm{SnO}_{2}$ & FA & 19 & - & 2 & - & - & 1 & - & 3 \\
\hline none & FA & 1 & - & - & - & - & - & - & - \\
\hline$(\mathrm{Sn})_{\mathrm{SSIE}}-$ beta1 & $1 \mathrm{BMF}$ & $(91)^{\mathrm{f}} 97$ & - & $(72)^{\mathrm{f}} 44$ & $(3)^{\mathrm{f}} 5$ & $(1)^{\mathrm{f}} 2$ & $(10)^{\mathrm{f}} 15$ & $(2)^{\mathrm{f}} 4$ & $(88)^{\mathrm{f}} 70$ \\
\hline$(\mathrm{Sn})_{\mathrm{SSIE}}$-beta1 & EMF & 98 & - & 52 & $5^{\mathrm{f}}$ & 2 & 17 & 4 & 80 \\
\hline deAl-beta1 & EMF & 91 & - & 72 & 9 & 1 & 7 & - & 89 \\
\hline H-beta & EMF & 100 & - & - & $15^{\mathrm{g}}$ & 16 & 15 & 1 & 47 \\
\hline $\mathrm{SnO}_{2}$ & EMF & 17 & - & 1 & $<1$ & - & 1 & $<1$ & 2 \\
\hline none & EMF & 13 & - & $<1$ & $<1$ & - & 2 & - & 2 \\
\hline$(\mathrm{Sn})_{\mathrm{SSIE}}$-beta & $\alpha A n L$ & $99^{\mathrm{h}}$ & - & - & $19^{\mathrm{h}}$ & $73^{h}$ & - & $2^{\mathrm{h}}$ & $94^{\mathrm{h}}$ \\
\hline deAl-beta1 & $\alpha A n L$ & $42^{\mathrm{h}}$ & - & - & $8^{\mathrm{h}}$ & $21^{\mathrm{h}}$ & - & - & $29^{\mathrm{h}}$ \\
\hline H-beta & $\alpha A n L$ & $100^{\mathrm{h}}$ & - & - & $15^{\mathrm{h}}$ & $51^{\mathrm{h}}$ & - & $2^{\mathrm{h}}$ & $68^{\mathrm{h}}$ \\
\hline $\mathrm{SnO}_{2}$ & $\alpha A n L$ & $9^{h}$ & - & - & $2^{\mathrm{h}}$ & - & - & $1^{\mathrm{h}}$ & $3^{h}$ \\
\hline None & $\alpha A n L$ & $13^{h}$ & - & - & $2^{\mathrm{h}}$ & - & - & - & $2^{\mathrm{h}}$ \\
\hline$(\mathrm{Sn})_{\mathrm{SSIE}}-\mathrm{beta} 1$ & LA & $44(68)^{i}$ & - & - & $10(19)^{\mathrm{i}}$ & - & $2(1)^{\mathrm{i}}$ & $6(25)^{\mathrm{i}}$ & $18(45)^{\mathrm{i}}$ \\
\hline deAl-beta1 & LA & 37 & - & - & 10 & - & 2 & - & 12 \\
\hline H-beta & LA & 75 & - & - & 52 & - & 2 & 4 & 58 \\
\hline $\mathrm{SnO}_{2}$ & LA & 33 & - & - & 11 & - & - & - & 11 \\
\hline None & LA & 23 & - & - & 9 & - & - & - & 9 \\
\hline \multirow[t]{2}{*}{$(\mathrm{Sn})_{\mathrm{SSIE}^{-}}-\mathrm{beta} 1$} & $1 \mathrm{BL}$ & $66(89)^{j}$ & - & - & - & - & - & $55(73)^{\mathrm{j}}$ & $55(73)^{\mathrm{j}}$ \\
\hline & $1 \mathrm{BL}^{\mathrm{k}}$ & $36^{\mathrm{k}}$ & - & - & - & - & - & $19^{\mathrm{k}}$ & $19^{\mathrm{k}}$ \\
\hline$(\mathrm{Sn})_{\mathrm{SSIE}}-\mathrm{beta} 2$ & $1 \mathrm{BL}$ & 28 & - & - & - & - & - & 2 & 2 \\
\hline
\end{tabular}




\begin{tabular}{|c|c|c|c|c|c|c|c|c|c|}
\hline$(\mathrm{Sn})_{\mathrm{SSIE}}$-beta3 & $1 \mathrm{BL}$ & 16 & - & - & - & - & - & 1 & 1 \\
\hline$(\mathrm{SnAl})_{\mathrm{SSIE}}$-beta1 & $1 \mathrm{BL}$ & 25 & - & - & 1 & 3 & - & 1 & 5 \\
\hline deAl-beta1 & $1 \mathrm{BL}$ & 19 & - & - & $<1$ & - & - & - & $<1$ \\
\hline H-beta & $1 \mathrm{BL}$ & 22 & - & - & 1 & 3 & - & 1 & 5 \\
\hline $\mathrm{SnO}_{2}$ & $1 \mathrm{BL}$ & 18 & - & - & - & - & - & - & - \\
\hline none & $1 \mathrm{BL}$ & 18 & - & - & - & - & - & - & - \\
\hline$(\mathrm{Sn})_{\mathrm{SSIE}}$-beta1 & EL & $65(75)^{\mathrm{j}}$ & - & - & - & - & - & $35(48)^{\mathrm{j}}$ & $35(48)^{\mathrm{j}}$ \\
\hline$(\mathrm{Sn})_{\mathrm{SSIE}}-\mathrm{beta} 2$ & EL & 26 & - & - & - & - & - & 1 & 1 \\
\hline$(\mathrm{Sn})_{\mathrm{SSIE}}$-beta3 & EL & 21 & - & - & - & - & - & $<1$ & $<1$ \\
\hline$(\mathrm{SnAl})_{\mathrm{SSIE}^{-b e t a} 1}$ & EL & 32 & - & - & - & - & - & $<1$ & $<1$ \\
\hline deAl-beta1 & EL & 14 & - & - & - & - & - & - & - \\
\hline H-beta & EL & 32 & - & - & 1 & - & - & 2 & 3 \\
\hline $\mathrm{SnO}_{2}$ & EL & 24 & - & - & - & - & - & - & - \\
\hline none & EL & 27 & - & - & - & - & - & - & - \\
\hline
\end{tabular}

${ }^{\mathrm{a}}$ Reaction conditions: $[\mathrm{S}]_{0}=0.45 \mathrm{M}$ in $2 \mathrm{BuOH}$, catalyst load $=27 \mathrm{~g}_{\text {cat }} \mathrm{L}^{-1}, 120^{\circ} \mathrm{C}$. ${ }^{\mathrm{b}}$ Substrate conversion at $24 \mathrm{~h}$ reaction, unless otherwise specified. ${ }^{\mathrm{c}}$ Total yield of bio-products at $24 \mathrm{~h}$ reaction, unless otherwise specified. ${ }^{\mathrm{d}}$ Catalytic results at $5 \mathrm{~h}$ reaction. ${ }^{\mathrm{e}}$ Catalytic results at $1 \mathrm{~h}$ reaction. ${ }^{\mathrm{f}}$ EL was formed in $1 \% .{ }^{\mathrm{g}}$ EL was formed in $4 \%{ }^{\mathrm{h}}$ Catalytic results at $7 \mathrm{~h}$ reaction. ${ }^{\mathrm{i}}$ Catalytic results at $72 \mathrm{~h}$ reaction ${ }^{\mathrm{j}}$ Catalytic results at $48 \mathrm{~h}$ reaction. ${ }^{\mathrm{k}}$ Water was added in an initial molar ratio $\mathrm{H}_{2} \mathrm{O}: 1 \mathrm{BL}=0.5$.

As referred above, of the ( $\mathrm{Sn}, \mathrm{Al})$-containing catalysts, $(\mathrm{Sn})_{\mathrm{SSIE}^{-}}$-beta1 was by far the bestperforming in the one-pot conversion of Fur ( $83 \%$ bio-products yield at $86 \%$ conversion, $5 \mathrm{~h}$ reaction, Table 3). The remaining ( $\mathrm{Sn}, \mathrm{Al}$ )-containing materials led to $10-17 \%$ bio-products total yield at 26-36\% conversion, 24 h. Poor catalytic effects were also observed for the Snfree materials deAl-betan and the parent H-beta zeolite (2-10\% yield at 22-43\% conversion, 24 h). Hence, Al-sites with Brönsted or Lewis behaviour (Table 2) were poorly effective for Fur conversion.

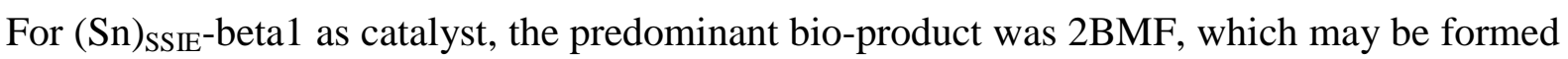
via consecutive CTH (Fur-to-FA) and acid-catalysed etherification (FA-to-2BMF) reactions 
[4]. The predominance of the furfuryl alkyl ether bio-product somewhat parallels that reported in the literature for the conversion of HMF (a relative of Fur) in the presence of (Alfree) Sn-beta, in alcohol media, at $120^{\circ} \mathrm{C}$, which gave mainly 2,5-bis(alkoxymethyl)furan products [114]. The Fur and HMF reaction systems involved the reduction of the furanic aldehyde group of the substrate (via $\mathrm{CTH}$ ), and subsequent acid-catalysed etherification (giving different products). The Sn-sites of the (Al-free) Sn-beta catalyst were effective in the two types of reactions of the HMF conversion [114]. In a parallel fashion, $(\mathrm{Sn})_{\mathrm{SSIE}^{-}}$beta1 may possesses Sn-sites which are effective in promoting the consecutive CTH and etherification reactions Fur-FA-2BMF. According to the literature, CTH reactions can be promoted by Snsites in tetrahedral coordination $[71,72,114,115]$ and, on the other hand, etherification reactions can be promoted by tetrahedral Sn- or Al-sites $[4,53,113,114,116,117]$. Hence, tetrahedral Sn-sites are important for initialising the overall process, i.e. with Fur reduction. For the best performing catalyst $(\mathrm{Sn})_{\mathrm{SSIE}}$-beta1, the characterisation studies indicated enhanced incorporation of (tetrahedrally coordinated) Sn-sites into the extensively dealuminated framework of deAl-beta1, and acidity essentially associated with (Lewis acid) Sn-sites. The poorer catalysts $(\mathrm{Sn})_{\mathrm{SSIE}}$-beta2 and $(\mathrm{Sn})_{\mathrm{SSIE}}$-beta3 possessed much lower amounts of Sn-sites than $(\mathrm{Sn})_{\mathrm{SSIE}}$-beta1.

It has been reported in the literature for $\mathrm{CTH}$ reactions between alcohols and ketones, in the presence of Sn-containing zeolites, that not all tetrahedral Sn-sites may be involved in the catalytic process, since those which are effectively active likely possess a hydrolysed $\mathrm{Sn}-\mathrm{O}-\mathrm{Si}$ bond [72, 114]. Accordingly, turnover frequencies calculated on the basis of total concentration of Sn-sites may lead to erroneous conclusions. On the other hand, cooperative effects of different active sites may occur, which makes it difficult to isolate the catalytic contributions of different metal sites in multifunctional catalysts. Thus, the contributions of different types of active sites of the prepared materials are discussed considering reactivity 
trends observed in the systematic catalytic studies, comparing the modified beta materials with or without Sn; since all modified beta materials were prepared from the same (nanocrystalline) H-beta sample, and possess comparable textural properties, the differences in catalytic performances may be considered to be essentially due to the surface chemical properties of the catalysts.

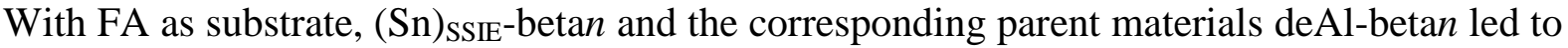
roughly comparable total yields of bio-products at $24 \mathrm{~h}$ reaction $(42-58 \%)$, Table 3 . The bioproducts formed were 2BMF (predominant), 2BL, AnLs and LA; GVL was only formed in the presence of $(\mathrm{Sn})_{\mathrm{SSIE}}$-beta1. Comparison of these catalytic results to those for Fur as substrate, suggests that while Al-sites were ineffective in the initial CTH step of the Fur conversion, they played roles in the subsequent acid-catalysed steps of FA to the bio-products 2BMF, 2BL, LA and AnLs. On the other hand, the reaction of FA was faster for $(\mathrm{Sn})_{\mathrm{SSIE}^{-}}$ beta1 than deAl-beta1, suggesting that the Sn-sites (besides Al-sites) of (Sn) SSIE-beta1 contributed to the FA conversion.

To the best of our knowledge, this is the first report of the etherification of FA to FEs using dealuminated zeolite beta catalysts. Lange et al.[118] reported comparable FE yields for the reaction of FA with ethanol, in the presence of zeolite $\mathrm{H}-\mathrm{ZSM}-5$, at $125{ }^{\circ} \mathrm{C}(50 \%$ maximum yield); AnLs, LA and the corresponding LE were formed with less than $10 \%$. Dumesic et al. [21] reported the conversion of FA to LA, in the presence of zeolite ZSM-5, in water/secbutylphenol solvent systems, at $120^{\circ} \mathrm{C}$, which gave $15 \%$ LA yield at $1 \mathrm{~h}$. The reaction of FA in the presence of $\mathrm{H}$-beta gave mainly to $2 \mathrm{BL}$, LA (14\% at $24 \mathrm{~h}$ reaction) and AnLs (Table $3)$.

For all modified catalysts, the reaction of Fur was slower than that of FA as substrate (Table 3), and, on the other hand, FA was very reactive under catalytic conditions, as evidenced by

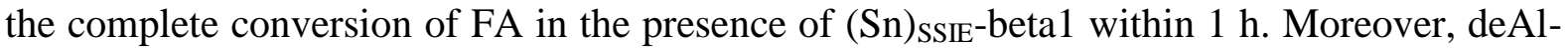


betan materials with very low total amounts of $(\mathrm{L}+\mathrm{B})$ acid sites (Table 2) promoted fairly well the reaction of FA. Hence, FA is much more reactive than Fur, which can explain the low concentrations of FA in the Fur reaction system (Table 3). The much higher reactivity of FA than Fur, together with the lower total yield of bio-products reached for FA as substrate, suggests that side reactions of FA are favoured for mixtures that are more concentrated in FA, which is in agreement with the literature [119-122].

The reactions of the FE substrates EMF and 1BMF, in the presence of $(\mathrm{Sn})_{\mathrm{SSIE}}$-beta1, gave mainly $2 \mathrm{BMF}$ (e.g. $72 \%$ 2BMF yield at 91\% 1BMF conversion), Table 3. These results indicate that $(\mathrm{Sn})_{\mathrm{SSIE}}$-beta1 possessed good catalytic activity for the transetherification of the FEs with $2 \mathrm{BuOH}$, to give $2 \mathrm{BMF}$. After $24 \mathrm{~h}$ reaction, the conversion of the FE substrates was ca. 98\%, and the bio-products distribution consisted of 2BMF (44-52\% yield), AnLs (15$17 \%$ ) and, to lower extents, $2 \mathrm{BL}$, LA and GVL (each with $\leq 5 \%$ yield). The conversions of the FE substrates to the corresponding LEs were not favoured (i.e. EMF to ethyl levulinate (EL), and 1BMF to 1-butyl levulinate (1BL)).

With EMF as substrate, the catalysts $(\mathrm{Sn})_{\mathrm{SSIE}^{-}}$-beta1 and deAl-beta1 led to similar bioproducts distributions, excluding GVL which was only formed in the former case (Table 3). On the other hand, the reaction of EMF was slightly faster for $(\mathrm{Sn})_{\mathrm{SSIE}}$-beta1 than deAl-beta1. These results are consistent with those for FA as substrate in that the Al- and Sn-sites of $(\mathrm{Sn})_{\mathrm{SSIE}}$-beta1 play roles in the acid-catalysed etherification reaction of FA. The reaction of EMF in the presence of zeolite H-beta gave mainly 2BL, LA and AnLs. In particular, LA and 2BL were formed with higher yields (15 and 16\%, respectively, at $24 \mathrm{~h}$ ) in the presence of $\mathrm{H}$ beta than its modified versions. These results correlated with the higher total amount of $(\mathrm{B}+\mathrm{L})$ acid sites of H-beta (Table 2), suggesting that the ring-opening reactions of FEs to 2BL and LA are favoured by enhanced acidity associated with Al-sites. 
The reaction of $\alpha \mathrm{AnL}$ in the presence of $(\mathrm{Sn})_{\mathrm{SSIE}^{-}}$beta1, gave mainly LA (73\% yield, at $99 \%$ conversion, $7 \mathrm{~h}$ ), together with $2 \mathrm{BL}$ and GVL (Table 3). Hence, LA was formed via the intermediate formation of AnLs. Similar bio-products spectrum was observed for deAl-beta1, excluding GVL which was not formed in the presence of this catalyst. These results indicate that Sn- and Al-sites are capable of promoting the acid-catalysed conversion of AnLs-to-(LA, 2BL). The material $(\mathrm{Sn})_{\mathrm{SSIE}^{-}}$-beta1 was much more active than deAl-beta1, which correlates with the higher total amount of acid sites (mainly Sn-sites) of the former (Table 2).

Manzer [117] claimed the acid-catalysed conversion of AnLs to LEs, in alcohol media, using different types of solid acid catalysts. One of the best examples reported in that invention was that of the reaction of $\alpha \mathrm{AnL}$ with $1 \mathrm{BuOH}$, in the presence of commercial Amberlyst- $15^{\mathrm{TM}}$, a well-known Brönsted solid acid, which gave 96\% 1BL yield at 99\% $\alpha$ AnL conversion, $1 \mathrm{~h}$, $100{ }^{\circ} \mathrm{C}$ [117]. The reaction of $\alpha \mathrm{AnL}$ was faster in the presence of $\mathrm{H}$-beta (100\% conversion at $7 \mathrm{~h}$ ) than deAl-beta1 (42\% conversion), with LA and 2BL as main bio-products.

The reaction starting from LA in the presence of $(\mathrm{Sn})_{\mathrm{SSIE}}-$ beta1 gave mainly $2 \mathrm{BL}$ and GVL, albeit slowly (19\% 2BL and 6\% GVL yield, at $44 \%$ conversion, $24 \mathrm{~h})$, Table 3 . The LA conversions for the Sn-free material deAl-beta1 and its $\mathrm{Sn}$-containing version $(\mathrm{Sn})_{\mathrm{SSIE}}$-beta1 were roughly comparable (37-44\%), which correlates with their somewhat comparable molar ratios $\mathrm{Si} / \mathrm{Al}$ (553-591) and amounts of Brönsted acid sites (Table 2), suggesting that the step LA-2BL was essentially promoted by the Brönsted acid Al-sites of (Sn) $)_{S S I E}$-beta1. The fact that deAl-beta1 and $(\mathrm{Sn})_{\mathrm{SSIE}^{-}}$beta1 possess very small amounts of Brönsted acid sites can at least partly explain the slow reaction of LA using these catalysts. Zeolite H-beta led to faster conversion of LA, leading to far higher 2BL yields (52\% 2BL yield, at 75\% conversion, 24 h), than its modified versions, likely due to much higher Brönsted acidity of H-beta (Table 2). On the other hand, GVL was formed in the case of $(\mathrm{Sn})_{\mathrm{SSIE}}$-beta1 and not of its parent material deAl-beta1, indicating the Sn-sites are important for GVL formation. Based on these 
results, $(\mathrm{Sn})_{\mathrm{SSIE}}$-beta1 performs as multifunctional catalyst with the contributions of both Aland Sn-sites to the one-pot Fur conversion.

With LEs as substrates and $(\mathrm{Sn})_{\mathrm{SSIE}}$-beta1 as catalyst, GVL was the only bio-product formed (Table 3). The best-performing catalyst was by far $(\mathrm{Sn})_{\mathrm{SSIE}}$-beta1; e.g. the reaction of $1 \mathrm{BL}$ gave $73 \%$ GVL yield at $89 \%$ conversion, whereas the remaining materials led to less than $2 \%$ GVL yield. The outstanding catalytic performance of $(\mathrm{Sn})_{\mathrm{SSIE}}$-beta1 parallels that observed for the Fur system. The conversion of Fur-to-FA, as well as that of LEs-to-GVL involve CTH reactions. Based on the material characterisation studies, and the ineffectiveness of $\mathrm{SnO}_{2}$ as catalyst in all (acid and reduction) steps of the overall process, the superior performance of $(\mathrm{Sn})_{\mathrm{SSIE}}$-beta1 is attributed to the enhanced incorporation of tetrahedral $\mathrm{Sn}$-sites in this material.

A comparative study for $(\mathrm{Sn})_{\mathrm{SSIE}}$-beta1 with the different substrates, indicated that GVL was formed with far higher yields from LEs (up to $73 \%$ yield) than from the remaining substrates ( $\leq 6 \%$ GVL yield). Hence, the favourable formation of $2 \mathrm{BL}$ in the one-pot conversion of Fur seems desirable for the enhanced formation of GVL. However, based on the catalytic results for $(\mathrm{Sn})_{\mathrm{SSIE}}$-beta1 with different substrates (e.g. FA and FEs) the formation of 2BL seemed

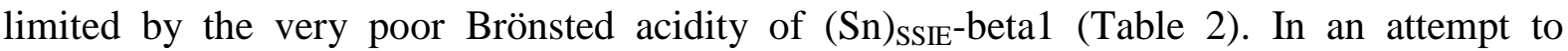
enhance the catalyst acidity, deAl-betal was subjected to SSIE for $\mathrm{Sn}$ and Al, giving $(\mathrm{SnAl})_{\mathrm{SSIE}}$-beta1. However, this material did not lead to improved catalytic results for the Fur conversion (Table 3$)$. The characterisation studies indicated that ( $\mathrm{SnAl})_{\mathrm{SSIE}^{-}}$-beta1 possessed enhanced acidity mainly associated with Al-sites, and relatively low amount of Sn-sites (Table 2), and heterogeneous Sn dispersion, accounting for the poor catalytic performance in Fur reduction (the first step of the reaction network).

Water is a co-product of some steps of the Fur conversion process and may influence the catalytic performance. The cyclisation of the LEs to GVL does not involve formation of 
water [123]. Hence, this step is somewhat interesting for investigating the influence of water on the catalytic activity. With $1 \mathrm{BL}$ as substrate and $(\mathrm{Sn})_{\mathrm{SSIE}}-$ beta1 as catalyst, the addition of a small amount of water (initial molar ratio of $\mathrm{H}_{2} \mathrm{O}: 1 \mathrm{BL}$ equal to 0.5 ) led to GVL as the only bio-product, albeit considerably affected the reaction rate; $1 \mathrm{BL}$ conversion at $24 \mathrm{~h}$ decreased from $66 \%$ to $36 \%$ (Table 3 ). Hence, inhibitory water effects may contribute to the low GVL yields reached in the overall process.

Overall, in terms of multifunctional catalytic properties, the tetrahedral Sn-sites are essential for the CTH steps, particularly of Fur to FA, initialising the one-pot reaction process, for which the Al-sites are ineffective. On the other hand, the Al- and Sn-sites are both involved in the acid-catalysed steps of FA-2BMF, 2BMF-(AnLs+2BL+LA) and AnLs-(LA+2BL), whereas the Al-sites are responsible for the step LA-2BL.

\subsubsection{Mechanistic proposal and kinetic modelling}

A comparative study of the bio-products distributions for all tested substrates (Fur, FEs, FA, $\alpha \mathrm{AnL}$ and LA) using $(\mathrm{Sn})_{\mathrm{SSIE}}$-beta1 as catalyst, suggested that the one-pot conversion of Fur involves the series of steps Fur-FA-2BMF-AnLs-LA-2BL-GVL (Scheme 2). The steps FAFE- $\alpha$ AnL-LE (discarding LA) have been contemplated in the mechanistic proposal by Zhang et al. [124] for the acid-catalysed conversion of FA in alcohol medium.

With the substrates Fur, FA and FEs and $(\mathrm{Sn})_{\mathrm{SSIE}}$-beta1 as catalyst, the initial concentration of 2BL tended to be higher than that of LA (Figure 9, Table 3). However, with $\alpha$ AnL as substrate the yields of LA were far higher than those of $2 \mathrm{BL}$, and, on the other hand, with the substrate LA gave $2 \mathrm{BL}$. These results suggest that $2 \mathrm{BL}$ is formed from Fur via parallel pathways with or without the intermediate formation of LA. According to the literature, the conversion of Fur to LEs may involve the intermediate formation of FA [125], FEs (formed 
from FA) $[4,118,119,124,126-128]$ or $\alpha A n L[129,130]$. Based on these mechanistic considerations, and the reactivity trends observed for $(\mathrm{Sn})_{\mathrm{SSIE}}$-beta1, the overall process may involve the conversion of $2 \mathrm{BMF}$ to $2 \mathrm{BL}$ with or without the intermediate formation of AnLs (Scheme 2).

Water is formed in the etherification of FA to FEs. It has been reported in the literature that in the presence of water and an acid catalyst, FA can be converted to LA [21], and, on the other hand, $\alpha A n L$ can be converted to LA [131]. Accordingly, the overall reaction of Fur may involve the conversion of FA to AnLs with or without the intermediate formation of FEs (Scheme 2). This hypothesis enters into consideration with mechanistic aspects reported by Khusnutdinov et al. [129] for the acid-catalysed conversion of FA in alcohol media, with the series of steps FA- $\alpha$ AnL-LEs taking place without necessarily involving the intermediate formation of FEs.

Based on the proposed mechanism for the overall process (Scheme 2), a kinetic model was developed as described in section 2.4. Possible loss-reactions of the species involved were considered, since, in general, the mole balances did not close for different bio-products as substrates. Figure 9 shows the calculated kinetic profiles for a $7 \mathrm{~h}$ batch reaction of Fur, in the presence of $(\mathrm{Sn})_{\mathrm{SSIE}^{-}}$-beta1, and the apparent kinetic rate constants $\left(k_{j}\right)$ are given in Table 4. The kinetic model fitted reasonably well the experimental data $\left(F_{\text {obj }}=2.75 \times 10^{-3}\right)$.

The model predicts slower conversion of Fur to FA $\left(k_{1}\right)$ than of FA to the bio-products 2BMF $\left(k_{2}\right)$ and AnLs $\left(k_{3}\right)$, which is consistent with the reactivity trends observed with Fur and FA as substrates, with the Sn-sites promoting the step Fur-FA, and both Al- and Sn-sites promoting the subsequent FA conversion. Based on the calculated apparent rate constants, the fastest step of the overall process was the conversion of FA to $2 \mathrm{BMF}\left(k_{2}\right)$, which is consistent with the fact that $2 \mathrm{BMF}$ was predominant bio-product in the reactions starting from Fur and FA. The bio-product 2BMF seems to be converted faster to AnLs $\left(k_{4}\right)$ than to $2 \mathrm{BL}\left(k_{5}\right)$, consistent 
with the reactivity trends observed with FEs as substrates. The formation of $2 \mathrm{BL}$ seems limited by the relatively slow LA-to-2BL $\left(k_{7}\right)$ and 2BMF-to-2BL $\left(k_{5}\right)$ steps. The step LA-2BL seems to limit the pathway of $2 \mathrm{BMF}$ to $2 \mathrm{BL}$ via AnLs since $k_{7}$ is the lowest of the kinetic constants for the pathway 2BMF-AnLs-LA-2BL. These results are consistent with the reactivity trends observed with FEs, $\alpha$ AnL and LA as substrates, where LA was the least reactive; specifically, the step LA-2BL was essentially promoted by the Brönsted Al-sites, present in very small amounts in $(\mathrm{Sn})_{\mathrm{SSIE}^{-}}$-beta1, whereas the acid-catalysed conversions of FE and $\alpha$ AnL were promoted by both the Al- and (far more abundant) Lewis acid Sn-sites. In summary, based on the observed reactivity trends for the systematic catalytic studies, together with the acid properties measurements, the rate limiting steps of $2 \mathrm{BL}$ formation seem to be associated with the very poor Brönsted acidity of $(\mathrm{Sn})_{\mathrm{SSIE}}$-beta1.

Table 4. Kinetic constants $\left(k_{j}\right)$, confidence intervals at $90 \%$ and parameter error $(\%)$, of the modelled overall reaction of Fur, in the presence of $(\mathrm{Sn})_{\mathrm{SSIE}}$-beta1, in 2-butanol, at $120{ }^{\circ} \mathrm{C}$.

\begin{tabular}{|c|c|c|}
\hline & Kinetic constants $\left(\mathrm{L} \mathrm{g} \mathrm{cat}^{-1} \mathrm{~h}^{-1}\right)$ & Error (\%) \\
\hline$k_{1}$ & $1.883 \times 10^{-2} \pm 0.070 \times 10^{-2}$ & 3.74 \\
\hline$k_{2}$ & $2.066 \pm 0.080$ & 13.13 \\
\hline$k_{3}$ & $3.059 \times 10^{-1} \pm 0.402 \times 10^{-1}$ & 3.18 \\
\hline$k_{4}$ & $2.694 \times 10^{-3} \pm 0.086 \times 10^{-3}$ & 0.79 \\
\hline$k_{5}$ & $8.069 \times 10^{-4} \pm 0.064 \times 10^{-4}$ & 12.26 \\
\hline$k_{6}$ & $1.856 \times 10^{-3} \pm 0.228 \times 10^{-3}$ & 8.82 \\
\hline$k_{7}$ & $2.341 \times 10^{-5} \pm 2.06 \times 10^{-6}$ & 2.87 \\
\hline$k_{8}$ & $1.368 \times 10^{-3} \pm 0.039 \times 10^{-3}$ & 12.28 \\
\hline$k_{9}$ & $7.416 \times 10^{-8} \pm 0.911 \times 10^{-8}$ & \\
\hline
\end{tabular}




\begin{tabular}{|c|c|c|}
\hline$k_{10}$ & $1.112 \times 10^{-7} \pm 0.095 \times 10^{-7}$ & 8.56 \\
\hline$k_{11}$ & $5.648 \times 10^{-8} \pm 0.792 \times 10^{-8}$ & 14.03 \\
\hline$k_{12}$ & $4.877 \times 10^{-3} \pm 0.637 \times 10^{-3}$ & 13.06 \\
\hline$k_{13}$ & $1.988 \times 10^{-3} \pm 0.076 \times 10^{-3}$ & 3.81 \\
\hline$k_{14}$ & $4.526 \times 10^{-3} \pm 0.367 \times 10^{-3}$ & 8.10 \\
\hline
\end{tabular}

Based on the above results, the overall process poses requirements on the catalyst in terms of $\mathrm{CTH}$ activity for initialising the multistep process, and, on the other hand, enhanced acidity for obtaining the more end bio-product 2BL. By comparing the apparent rate constants of the two CTH steps Fur-to-FA and 2BL-to-GVL, the catalyst seems more efficient in the former reduction.

The model predicts slightly faster conversion of Fur than that observed experimentally after ca. $5 \mathrm{~h}$ reaction, which may be partly due to increasing importance of catalyst deactivation due to coking and water effects.

\section{Conclusions}

The one-pot conversion of furfural (Fur) to useful bio-products, namely furfuryl alkyl ethers (FEs), levulinate esters (LEs), levulinic acid (LA), angelica lactones (AnLs) and $\gamma$ valerolactone (GVL), was investigated using a heterogeneous inorganic catalyst, in 2-butanol, at $120^{\circ} \mathrm{C}$. Different catalytic materials which consisted of modified versions of zeolite beta containing $\mathrm{Al}$ and $\mathrm{Sn}$ sites, were prepared from commercially available nanocrystaline zeolite beta via post-synthesis partial dealumination followed by solid-state ion-exchange. The material with $\mathrm{Si} /(\mathrm{Al}+\mathrm{Sn})=553(\mathrm{Sn} / \mathrm{Al}=27.6)$ was the best performing catalyst $\left((\mathrm{Sn})_{\mathrm{SSIE}}\right.$-beta1 $)$, with $83 \%$ total yield of bio-products at $86 \%$ Fur conversion, and steady catalytic performance 
in six consecutive batch runs. The predominant bio-product was the FE. The superior catalytic performance of $(\mathrm{Sn})_{\mathrm{SSIE}}$-betal was attributed to homogeneous $\mathrm{Sn}$ dispersion and enhanced incorporation of (tetrahedral) Sn-sites into the extensively dealuminated deAl-beta1 parent material. These catalyst features were decisive for efficiently initialising the multistep conversion of Fur (i.e. reduction of Fur over Sn-sites), and, on the other hand, for the reduction of the LE to GVL. On the other hand, the Sn-sites and the Al-sites of the multifunctional catalysts played roles in acid-catalysed steps of FA-2BMF, 2BMF$(A n L s+2 B L+L A)$ and AnLs-(LA+2BL), whereas the Al-sites were mostly responsible for the step LA-2BL. The $(\mathrm{Sn})_{\mathrm{SSIE}}$-beta1 catalyst possessed poor Brönsted acidity for shifting the multistep process towards more-end bio-products, namely LEs. Hence, although this catalyst was effective for producing GVL in reactions starting from LEs, it led low GVL yields in the one-pot conversion of Fur. On the other hand, water is formed in the overall process, which negatively affected the catalytic performance in the LE-to-GVL conversion. Based on the mechanistic proposal for the overall process, a kinetic model was developed, with reasonably good fitting for the best performing catalyst, and kinetic constants consistent with experimentally observed reactivity trends (based on catalytic systematic studies).

The attempts to improve the catalytic performance towards formation of more end bioproducts were made by using less harsh dealumination conditions and introducing less amount of tin via SSIE (changing the $\mathrm{Sn} / \mathrm{Al}$ ratio). However, this led to heterogeneous $\mathrm{Sn}$ dispersion with reduced incorporation of Sn-sites into the framework, which accounted for poor catalytic performance in the overall process. All poor performing SnAl-containing beta materials were effective for acid-catalysed conversion of FA to bio-products. The challenge remains to establish the optimal conditions of the post-synthesis protocol and/or prepare multifunctional catalysts with good compromises between different types of active sites for targeting specific bio-products in the one-pot conversion of Fur. 


\section{Acknowledgements}

This work was developed in the scope of the project CICECO-Aveiro Institute of Materials (Ref. FCT UID /CTM /50011/2013), financed by national funds through the FCT/MEC and when applicable co-financed by FEDER under the PT2020 Partnership Agreement. The FCT and the European Union are acknowledged for grants to M.M.A. (SFRH/BPD/89068/2012), P.N. (SFRH/BPD/73540/2010), A.M. (SFRH/BPD/95393/2013) and SFRH/BPD/91397/2012, cofunded by MCTES and the ESF through the program POPH of QREN. The authors wish to thank António J. S. Fernandes (I3N, Department of Physics, University of Aveiro) for assistance with the UV-Raman data measurements; and Luís Mafra and Mariana Sardo for helpful discussions of NMR characterization. SL and AU thank MINECO for support through Severo Ochoa Excellence Accreditation 2014-2018 (SEV2013-0319).

\section{References}

[1] K.J. Zeitsch, The Chemistry and Technology of Furfural and Its Many By-Products, 1st ed ed., Elsevier Science B. V., Amesterdam, The Netherlands, 2000.

[2] M.J. Climent, A. Corma, S. Iborra, Green Chem.16 (2014) 516.

[3] P. Maeki-Arvela, B. Holmbom, T. Salmi, D.Y. Murzin, Catal. Rev. 49 (2007) 197.

[4] L. Bui, H. Luo, W.R. Gunther, Y. Román-Leshkov, Angew. Chem. Int. Ed. 52 (2013) 8022.

[5] P.S. Watson, J.A. Nuzum, D.B. Rohr, D.E. Newquist, C.T. Crawford, L.M. Bragg, in: R. A. Rogowsky (Eds), Furfuryl Alcohol from China, South Africa, and Thailand, U.S. International Trade Commission, Washington DC, 1994. 
[6] A.J.J.E. Eerhart, W.J.J. Huijgen, R.J.H. Grisel, J.C. Van der Waal, E. de Jong, A.D.S. Dias, A.P.C. Faaij, M.K. Patel, RSC Adv. 4 (2014) 3536.

[7] R.J. Haan, J.-P. Lange, US Patent 8372164 B2 (2013), to Shell Oil Company.

[8] B. Vanderhaegen, H. Neven, L. Daenen, K.J. Verstrepen, H. Verachtert, G. Derdelinckx, J. Agr. Food Chem. 52 (2004) 1661.

[9] AROXA ${ }^{\mathrm{TM}}$, 2-furfuryl ethyl ether. http://www.aroxa.com/discovery/discovery-standard/2furfuryl-ethyl-ether/, consulted in October 2014.

[10] H. Joshi, B.R. Moser, J. Toler, W.F. Smith, T. Walker, Biomass Bioenerg. 35 (2011) 3262.

[11] E.S. Olson, in: National Technical Information Service (Eds), SUBTASK 4.1Conversion of Lignocellulosic Material to Chemicals and Fuels, United States Government, Energy and [12] L.E. Manzer, WO Patent 2005097724 A1 (2005), to E. I. Du Pont de Nemours and Company.

[13] L. Lomba, B. Giner, I. Bandres, C. Lafuente, M. Rosa Pino, Green Chem. 13 (2011) 2062.

[14] H.J. Bart, J. Reidetschlager, K. Schatka, A. Lehmann, Ind. Eng. Chem. Res. 33 (1994) 21.

Environmental Research Center, University of North Dakota, Grand Forks, 2001.

[15] G.W. Huber, J.N. Chheda, C.J. Barrett, J.A. Dumesic, Science, 308 (2005) 1446.

[16] J.-P. Lange, R. Price, P.M. Ayoub, J. Louis, L. Petrus, L. Clarke, H. Gosselink, Angew. Chem. Int. Ed. 49 (2010) 4479.

[17] J.Q. Bond, D.M. Alonso, D. Wang, R.M. West, J.A. Dumesic, Science, 327 (2010) 1110.

[18] I.T. Horváth, H. Mehdi, V. Fabos, L. Boda, L.T. Mika, Green Chem. 10 (2008) 238.

[19] I.T. Horváth, in: 10th Annual Green Chemistry \& Engineering Conference (Eds), Designing for a Sustainable Future, Abstract number 27, Washington DC, 2006. 
[20] J.J. Bozell, L. Moens, D.C. Elliott, Y. Wang, G.G. Neuenscwander, S.W. Fitzpatrick, R.J. Bilski, J.L. Jarnefeld, Resour. Conserv. Recy. 28 (2000) 227.

[21] E.I. Gürbüz, S.G. Wettstein, J.A. Dumesic, ChemSusChem. 5 (2012) 383.

[22] H.-J. Ha, S.-K. Lee, Y.-J. Ha, J.-W. Park, Synthetic Commun. 24 (1994) 2557.

[23] R. E. Holmen. US Patent 3471554 (1969), to Minnesota Mining and Manufacturing Company.

[24] R. Cao, J. Xin, Z. Zhang, Z. Liu, X. Lu, B. Ren, S. Zhang, ACS Sustainable Chem. Engin. 2 (2014) 902.

[25] J. Cardellach, J. Font, R.M. Ortuno, J. Heterocyclic Chem. 21 (1984) 327.

[26] D.C. Elliott, J.G. Frye, US Patent 5883266 (1999), to Battelle Memorial Institute.

[27] D. Fegyverneki, L. Orha, G. Lang, I.T. Horvath,Tetrahedron, 66 (2010) 1078.

[28] W.R.H. Wright, R. Palkovits, ChemSusChem, 5 (2012) 1657.

[29] H. Mehdi, V. Fabos, R. Tuba, A. Bodor, L.T. Mika, I.T. Horvath, Top. Catal. 48 (2008) 49.

[30] J.-P. Lange, J.Z. Vestering, R.J. Haan, Chem. Commun. (2007) 3488.

[31] M. Chalid, H.J. Heeres, A.A. Broekhuis, J. Appl. Polymer Sci.123 (2012) 3556.

[32] R. Palkovits, Angew. Chem. Int. Ed. 49 (2010) 4336.

[33] J.C. Serrano-Ruiz, D. Wang, J.A. Dumesic, Green Chem.12 (2010) 574.

[34] J.C. Serrano-Ruiz, D.J. Braden, R.M. West, J.A. Dumesic, Appl. Catal. B: Environ. 100 (2010) 184.

[35] M. Vasiliu, K. Guynn, D.A. Dixon, J. Phys. Chem. C 115 (2011) 15686.

[36] E. Taarning, C.M. Osmundsen, X. Yang, B. Voss, S.I. Andersen, C.H. Christensen, Energ. Environ. Sci. 4 (2011) 793.

[37] G.W. Huber, A. Corma, Angew. Chem. Int. Ed. 46 (2007) 7184. 
[38] J. Gallo, D. Alonso, M. Mellmer, J. Yeap, H. Wong, J. Dumesic, Top. Catal. 56 (2013) 1775.

[39] M. Kaldström, N. Kumar, T. Salmi, D.Y. Murzin, Cell. Chem. Technol. 44 (2010) 203.

[40] M.Käldström, N. Kumar, T. Heikkilä, M. Tiitta, T. Salmi, D.Y. Murzin, ChemCatChem. $2(2010) 539$.

[41] S. Lima, M.M. Antunes, A. Fernandes, M. Pillinger, M.F. Ribeiro, A.A. Valente, Appl. Catal. A: Gen. 388 (2010) 141.

[42] L.R. Ferreira, S. Lima, P. Neves, M.M. Antunes, S.M. Rocha, M. Pillinger, I. Portugal, A.A. Valente, Chem. Engin. J. 215-216 (2013) 772.

[43] K.-I. Shimizu, R. Uozumi, A. Satsuma, Catal. Commun. 10 (2009) 1849.

[44] P. Dornath, W. Fan, Micropor Mesopor Mater. 191 (2014) 10.

[45] L. Hu, Z. Wu, J. Xu, Y. Sun, L. Lin, S. Liu, Chem. Engin. J. 244 (2014) 137.

[46] R. Otomo, T. Yokoi, J.N. Kondo, T. Tatsumi, Appl. Catal. A: Gen. 470 (2014) 318.

[47] P. Rivalier, J. Duhamet, C. Moreau, R. Durand, Catal. Today, 24 (1995) 165

[48] S. Saravanamurugan, A. Riisager, ChemCatChem. 5 (2013) 1754.

[49] S. Saravanamurugan, A. Riisager, Catal. Commun.17 (2012) 71.

[50] A. Corma, O. de la Torre, M. Renz, Energ. Environ. Sci. 5 (2012) 6328.

[51] L. Faba, B.T. Kusema, E.V. Murzina, A. Tokarev, N. Kumar, A. Smeds, E. Díaz, S. Ordóñez, P. Mäki-Arvela, S. Willför, T. Salmi, D.Y. Murzin, Micropor. Mesopor. Mater. 189 (2014) 189.

[52] R.M. West, M.S. Holm, S. Saravanamurugan, J. Xiong, Z. Beversdorf, E. Taarning, C.H. Christensen, J. Catal. 269 (2010) 122.

[53] P. Neves, S. Lima, M. Pillinger, S.M. Rocha, J. Rocha, A.A. Valente, Catal. Today, 218219 (2013) 76. 
[54] K.S. Arias, S.I. Al-Resayes, M.J. Climent, A. Corma, S. Iborra, ChemSusChem. 6 (2010) 123.

[55] K.S. Arias, M.J. Climent, A. Corma, S. Iborra, ChemSusChem. 7 (2014) 210.

[56] A. Aho, N. Kumar, A.V. Lashkul, K. Eränen, M. Ziolek, P. Decyk, T. Salmi, B. Holmbom, M. Hupa, D.Y. Murzin, Fuel 89 (2010) 1992.

[57] A. Aho, N. Kumar, K. Eränen, T. Salmi, M. Hupa, D.Y. Murzin, Fuel 87 (2008) 2493.

[58] M. Inaba, K. Murata, I. Takahara, Y. Liu, J. Chem. Engin. Japn. 47 (2014) 345.

[59] C.A. Mullen, A.A. Boateng, D.J. Mihalcik, N.M. Goldberg, Energ. Fuels, 25 (2011) 5444.

[60] D.L. Compton, M.A. Jackson, D.J. Mihalcik, C.A. Mullen, A.A. Boateng, J.Anal. Appl. Pyrol. 90 (2011) 174.

[61] E.M. Sulman, V.V. Alferov, Y.Y. Kosivtsov, A.I. Sidorov, O.S. Misnikov, A.E. Afanasiev, N. Kumar, D. Kubicka, J. Agullo, T. Salmi, D.Y. Murzin, Chem. Engin. J. 134 (2007) 162.

[62] T. Mochizuki, S.-Y. Chen, M. Toba, Y. Yoshimura, Appl. Catal. A: Gen. 456 (2013) 174.

[63] K. Murata, Y. Liu, M. Inaba, I. Takahara, J. Anal. Appl. Pyrol. 94 (2012) 75.

[64] K. Murata, P. Somwongsa, S. Larpkiattaworn, Y. Liu, M. Inaba, I. Takahara, Energ. Fuels, 25 (2011) 5429.

[65] A. Aho, A. Tokarev, P. Backman, N. Kumar, K. Eränen, M. Hupa, B. Holmbom, T. Salmi, D.Y. Murzin, Top. Catal. 54 (2011) 941.

[66] A. Aho, N. Kumar, K. ErÃănnen, T. Salmi, M. Hupa, D.Y. Murzin, Process Saf. Environ. Prot. 85 (2007) 473.

[67] N. Batalha, A.V. da Silva, M.O. de Souza, B.M.C. da Costa, E.S. Gomes, T.C. Silva, T.G. Barros, M.L.A. Gonçalves, E.B. Caramão, L.R.M. dos Santos, M.B.B. Almeida, 
R.O.M.A. de Souza, Y.L. Lam, N.M.F. Carvalho, L.S.M. Miranda, M.M. Pereira, ChemSusChem. 7 (2014) 1627.

[68] K. Giannakopoulou, M. Lukas, A. Vasiliev, C. Brunner, H. Schnitzer, Bioresource Technol. 101 (2010) 3209.

[69] Y. Yu, X. Li, L. Su, Y. Zhang, Y. Wang, H. Zhang, Appl. Catal. A: Gen. 447-448 (2012) 115.

[70] Z. Luo, S. Wang, X. Guo, J. Anal. Appl. Pyrol. 95 (2012) 112.

[71] A. Corma, M.E. Domine, L. Nemeth, S. Valencia, J. Am. Chem. Soc.124 (2002) 3194.

[72] R.S. Assary, L.A. Curtiss, J.A. Dumesic, ACS Catal. 3 (2013) 2694.

[73] C. Hammond, S. Conrad, I. Hermans, Angew. Chem. Int. Ed. 51 (2012) 11736.

[74] H.G. Karge, in: G. Ertl, H. Knözinger, F. Schüth, J. Weitkamp (Eds.), Solid-State Ion Exhange in Zeolites, Handbook of Heterogeneous Catalysis, Wiley-VCH Verlag GmbH \& Co., KGaA, 2008, pp. 484-510.

[75] C.J. Jia, P. Massiani, P. Beaunier, D. Barthomeuf, Appl. Catal. A: Gen.106 (1993) L185. [76] N. Kumar, P. Mäki-Arvela, S. Diáz, A. Aho, Y. Demidova, J. Linden, A. Shepidchenko, M. Tenhu, J. Salonen, P. Laukkanen, A. Lashkul, J. Dahl, I. Sinev, A.-R. Leino, K. Kordas, T. Salmi, D. Murzin, Top. Catal. 56 (2013) 696.

[77] J. Wittayakun, N. Grisdanurak, G. Kinger, H. Vinek, Korean J. Chem. Engin. 21 (2004) 950.

[78] C.E. Webster, R.S. Drago, M.C. Zerner, J. Am. Chem. Soc.120 (1998) 5509.

[79] R. O`Neill, M.N. Ahmad, L. Vanoye, F. Aiouache, Ind. Engin. Chem. Res. 48 (2009) 4300.

[80] J.M. Campelo, F. Lafont, J.M. Marinas, J. Chem. Soc. Faraday Trans 91 (1995).

[81] J.A. Egea, D. Henriques, T. Cokelaer, A.F. Villaverde, A. MacNamara, D.P. Danciu, J.R. Banga, J. Saez-Rodriguez, BMC Bioinformatics 15 (2014) 136. 
[82] Z. Kang, X. Zhang, H. Liu, J. Qiu, K.L. Yeung, Chem. Engin. J. 218 (2013) 425.

[83] R. Hajjar, Y. Millot, P.P. Man, M. Che, S. Dzwigaj, J. Phys. Chem. C 112 (2008) 20167.

[84] M. Renz, T. Blasco, A. Corma, V. Fornes, R. Jensen, L. Nemeth, Chem. Eur. J., 8 (2002) 4708.

[85] R. Baran, Y. Millot, T. Onfroy, J.-M. Krafft, S. Dzwigaj, Micropor. Mesopor. Mater. 163 (2012) 122.

[86] P. Li, G. Liu, H. Wu, Y. Liu, J.-g. Jiang, P. Wu, J. Phys. Chem. C 115 (2011) 3663.

[87] J. Janas, J. Gurgul, R.P. Socha, J. Kowalska, K. Nowinska, T. Shishido, M. Che, S. Dzwigaj, J. Phys. Chem. C 113 (2009) 13273.

[88] A. Mihaylova, K. Hadjiivanov, S. Dzwigaj, M. Che, J. Phys. Chem. B 110 (2006) 19530.

[89] S. Dzwigaj, M. Che, J. Phys. Chem. B 110 (2006) 12490.

[90] M. Zarrinkhameh, A. Zendehnam, S.M. Hosseini, N. Robatmili, M. Arabzadegan, Bull. Mater. Sci. 37 (2014) 533.

[91] J. Arbiol, E. Comini, G. Faglia, G. Sberveglieri, J.R. Morante, J. Cryst. Growth, 310 (2008) 253.

[92] P.S. Niphadkar, P.N. Joshi, S.S. Deshpande, V.V. Bokade, Appl. Catal. A: Gen. 401 (2011) 182.

[93] A. Corma, M.E. Domine, S. Valencia, J. Catal. 215 (2003) 294.

[94] K. Byrappa, B.V.S. Kumar, Asian J. Chem. 19 (2007) 4933.

[95] S.J. Kang, Y.J. Gong, T. Dou, Y. Zhang, Y.Y. Zheng, Pet. Sci., 4 (2007) 70.

[96] C. Li, Z. Wu, in: S.M. Auerbach, K.A. Carrado, P.K. Dutta (Eds.), Microporous Materials Characterized by Vibrational Spectroscopies, Handboook of Zeolite Science and Technology, CRC Press, Basel, Switzerland, 2003.

[97] P. Fejes, J.B. Nagy, K. Kovacs, G. Vanko, Appl. Catal. A: Gen. 145 (1996) 155. 
[98] M. A. Camblor, A. Corma, S. Valencia, J. Mater. Chem. 8 (1998) 2137.

[99] V.I. Parvulescu, S.M. Coman, N. Candu, J. El Haskouri, D. Beltran, P. Amoros, J. Mater. Sci. 44 (2009) 6693.

[100] K. Möller, B. Yilmaz, R.M. Jacubinas, U. Müller, T. Bein, J. Am. Chem. Soc. 133 (2011) 5284 .

[101] B. Mihailova, V. Valtchev, S. Mintova, A.C. Faust, N. Petkov, T. Bein, Phys. Chem. Chem. Phys. 7 (2005) 2756.

[102] W. Chen, D. Ghosh, S. Chen, J. Mater. Sci. 43 (2008) 5291.

[103] B. Tang, W. Dai, G. Wu, N. Guan, L. Li, M. Hunger, ACS Catal. 4 (2014) 2801.

[104] H.Y. Luo, L. Bui, W.R. Gunther, E. Min, Y. Roman-Leshkoy, ACS Catal. 2 (2012) 2695.

[105] M.P. Pachamuthu, K. Shanthi, R. Luque, A. Ramanathan, Green Chem. 15 (2013) 2158.

[106] J.A. Dumesic, R.M. West. US Patent 7960592 B1 (2011), to Wisconsin Alumni Research Foundation.

[107] T. Pasini, A. Lolli, S. Albonetti, F. Cavani, M. Mella, J. Catal.317 (2014) 206.

[108] P. Panagiotopoulou, N. Martin, D.G. Vlachos, J. Mol. Catal. A: Chem. 392 (2014) 223.

[109] P. Panagiotopoulou, D.G. Vlachos, Appl. Catal. A: Gen. 480 (2014) 17.

[110] M. Chia, J.A. Dumesic, Chem. Commun. 47 (2011) 12233.

[111] X. Tang, H. Chen, L. Hu, W. Hao, Y. Sun, X. Zeng, L. Lin, S. Liu, Appl. Catal. B: Environ. 147 (2014) 827.

[112] Z. Yang, Y.-B. Huang, Q.-X. Guo, Y. Fu, Chem. Commun. 49 (2013) 5328.

[113] J. Jae, E. Mahmoud, R.F. Lobo, D.G. Vlachos, ChemCatChem. 6 (2014) 508.

[114] J.D. Lewis, S. Van de Vyver, A.J. Crisci, W.R. Gunther, V.K. Michaelis, R.G. Griffin, Y. Roman-Leshkov, ChemSusChem, 7 (2014) 2255. 
[115] A. Corma, M. Renz, Angew. Chem. 119 (2007) 302.

[116] P. Neves, M.M. Antunes, P.A. Russo, J.P. Abrantes, S. Lima, A. Fernandes, M. Pillinger, S.M. Rocha, M.F. Ribeiro, A.A. Valente, Green Chem. 15 (2013) 3367.

[117] L.E. Manzer, WO Patent 2005097723 A2 (2005) to E. I. du Pont de Nemours and Company: Legal Patent Records Center.

[118] J.P. Lange, E. Van der Heide, J. Van Buijtenen, R. Price, ChemSusChem. 5 (2012) 150. [119] A.M. Hengne, S.B. Kamble, C.V. Rode, Green Chem. 15 (2013) 2540.

[120] T. Kim, R.S. Assary, C.L. Marshall, D.J. Gosztola, L.A. Curtiss, P.C. Stair, ChemCatChem. 3 (2011) 1451.

[121] F. Jing, S. Donen, S. Selifonov, B. Mullen, WO Patent 2010102203 A2 (2010) to Segetis: Sustainable Chemistry Solutions.

[122] D. Alonso, J. Bond, M. Chia, J. Dumesic, T. Root, WO Patent 2012162001 A1 (2012) to Wisconsin Alumni Research Foundation.

[123] J. Dědeček, L. Čapek, D. Kaucký, Z. Sobalík, B. Wichterlová, J. Catal. 211 (2002) 198. [124] Z. Zhang, K. Dong, Z. Zhao, ChemSusChem. 4 (2011) 112.

[125] B. Chen, F. Li, Z. Huang, T. Lu, Y. Yuan, G. Yuan, ChemSusChem. 7 (2014) 202.

[126] P. Demma Carà, R. Ciriminna, N.R. Shiju, G. Rothenberg, M. Pagliaro, ChemSusChem. 7 (2014) 835.

[127] L. Peng, H. Li, L. Xi, K. Chen, H. Chen, Bioresources, 9 (2014) 3825.

[128] G.M. Gonzalez Maldonado, R.S. Assary, J.A. Dumesic, L.A. Curtiss, Energ. Environ. Sci. 5 (2012) 8990.

[129] R.I. Khusnutdinov, A.R. Baiguzina, A.A. Smirnov, R.R. Mukminov, U.M. Whemilev, Russ. J. Appl. Chem. 80 (2007) 1687.

[130] D.M. Alonso, S.G. Wettstein, M.A. Mellmer, E.I. Gurbuz, J.A. Dumesic, Energ. Environ. Sci. 6 (2013) 76. 
[131] E.F. Mai, M.A. Machado, T.E. Davies, J.A. Lopez-Sanchez, V. Teixeira da Silva, Green Chem. 16 (2014) 4092. 


\section{SUPPLEMENTARY DATA}

Table S1. Catalytic performance of $(\mathrm{Sn})_{\mathrm{SSIE}}$-beta1 in the reactions of Fur and FA in $2 \mathrm{BuOH}$ or $2 \mathrm{PrOH} .^{\mathrm{a}}$

\begin{tabular}{|c|c|c|c|c|c|c|c|c|}
\hline \multirow{2}{*}{$\begin{array}{l}\text { Substrate } \\
\text { (reacting } \\
\text { solvent) }\end{array}$} & \multirow{2}{*}{$\begin{array}{c}\text { Conv. }^{\mathrm{b}} \text { at } \\
5 \mathrm{~h} / 24 \mathrm{~h} \\
(\%)\end{array}$} & \multicolumn{6}{|c|}{ Bio-product yield at 5/24 h (\%) } & \multirow{2}{*}{$\begin{array}{l}\text { Total bio- } \\
\text { products } \\
\text { yield }^{\mathrm{e}}(\%)\end{array}$} \\
\hline & & FA & $\mathrm{FE}^{\mathrm{c}}$ & $\mathrm{LE}^{\mathrm{d}}$ & LA & AnLs & GVL & \\
\hline Fur $(2 \mathrm{BuOH})$ & $86 / 95^{\mathrm{f}, \mathrm{h}}$ & $<1 / 1$ & $58 / 29$ & $4 / 11$ & $3 / 14$ & $18 / 23$ & $<1 / 2$ & $83 / 80$ \\
\hline Fur $(2 \mathrm{PrOH})$ & $37 / 79^{\mathrm{g}, \mathrm{h}}$ & $-/ 1$ & $7 / 14$ & $2 / 8$ & $-/ 4$ & $1 / 9$ & $-/-$ & $10 / 36$ \\
\hline $\mathrm{FA}(2 \mathrm{BuOH})$ & $100 /-^{f}$ & $-/-$ & $29 /-$ & $4 /-$ & $2 /-$ & $15 /-$ & $-/-$ & $51 /-$ \\
\hline $\mathrm{FA}(2 \mathrm{PrOH})$ & $97 /-^{\mathrm{g}}$ & $-1-$ & $29 /-$ & $4 /-$ & $1 /-$ & $13 /-$ & $-/-$ & $47 /-$ \\
\hline $1 \mathrm{BL}(2 \mathrm{BuOH})$ & $66^{\mathrm{f}}$ & - & - & - & - & - & 55 & 55 \\
\hline $1 \mathrm{BL}(2 \mathrm{PrOH})$ & $85^{\mathrm{g}}$ & - & - & - & - & - & 59 & 59 \\
\hline $1 \mathrm{EL}(2 \mathrm{BuOH})$ & $65^{\mathrm{f}}$ & - & - & - & - & - & 35 & 35 \\
\hline 1EL (2PrOH) & $81^{\mathrm{g}}$ & - & - & - & - & - & 35 & 35 \\
\hline
\end{tabular}

${ }^{\mathrm{a}}$ Reaction conditions: [Substrate $]_{0}=0.45 \mathrm{M}$ in aliphatic alcohol, catalyst load $=27 \mathrm{~g}_{\text {cat }} \mathrm{L}^{-1}, 120{ }^{\circ} \mathrm{C}$. ${ }^{\mathrm{b}}$ Substrate conversion at $24 \mathrm{~h}$ reaction. ${ }^{c}$ For the reaction in $2 \mathrm{BuOH}, \mathrm{FE}=2 \mathrm{BMF}=$ furfuryl 2-butyl ether, and for the reaction in $2 \mathrm{PrOH}, \mathrm{FE}=$ furfuryl 2-propyl ether. ${ }^{\mathrm{d}}$ For the reaction in $2 \mathrm{BuOH}, \mathrm{LE}=2 \mathrm{BL}=2-$ butyl levulinate, and for the reaction in $2 \mathrm{PrOH}, \mathrm{LE}=2$-propyl levulinate. ${ }^{\mathrm{e}}$ Total yield of bio-products at $24 \mathrm{~h}$ reaction. ${ }^{\mathrm{f}} 2 \mathrm{BuOH}$ as $\mathrm{H}$-donor. ${ }^{\mathrm{g}} 2 \mathrm{PrOH}$ as $\mathrm{H}$-donor. 

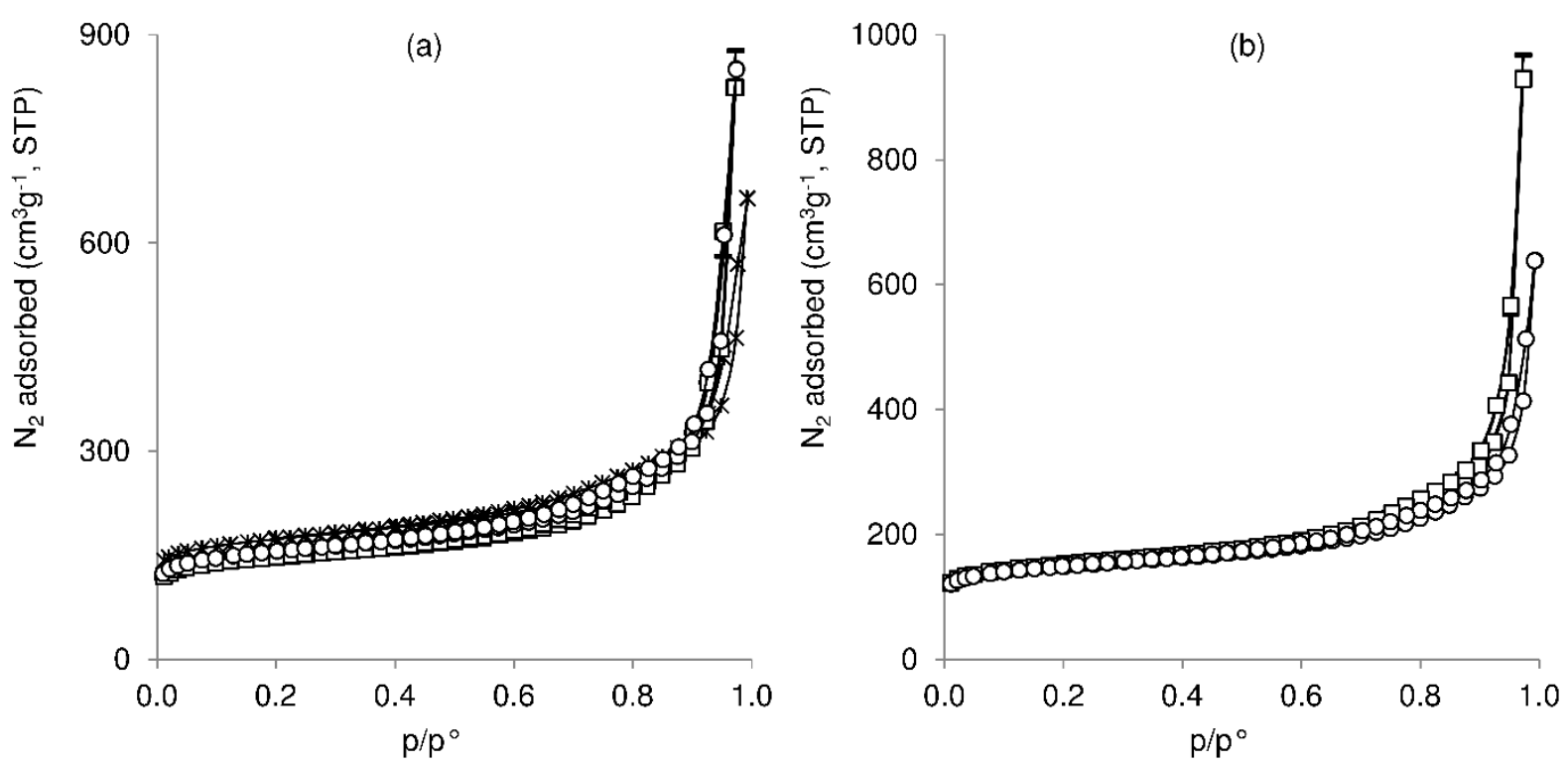

Figure S1. Nitrogen adsorption-desorption isotherms at $-196{ }^{\circ} \mathrm{C}$ for (a) H-beta $(*)$, deAl-

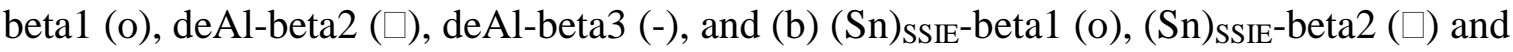
$(\mathrm{Sn})_{\mathrm{SSIE}^{-}}$beta3 (-); the symbols used in (a) and (b) are the same for each $n$ pair of materials deAl-betan and (Sn) SSIE $^{-b e t a n}$.

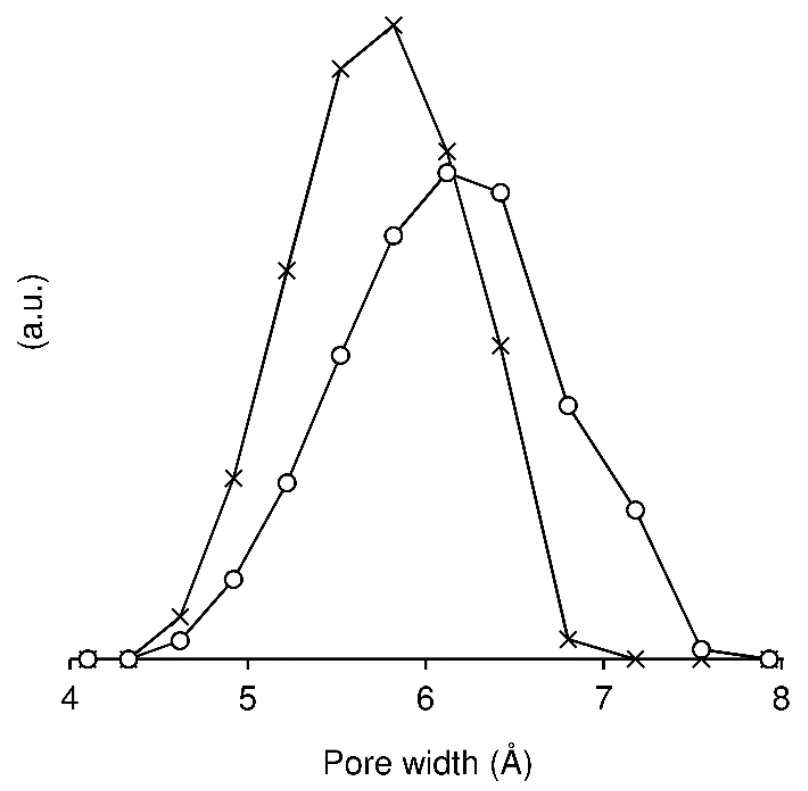

Figure S2. Micropore size distribution of deAl-beta1 $(\times)$ and $(\mathrm{Sn})_{\mathrm{SSIE}^{-}}$-beta1 (o). 


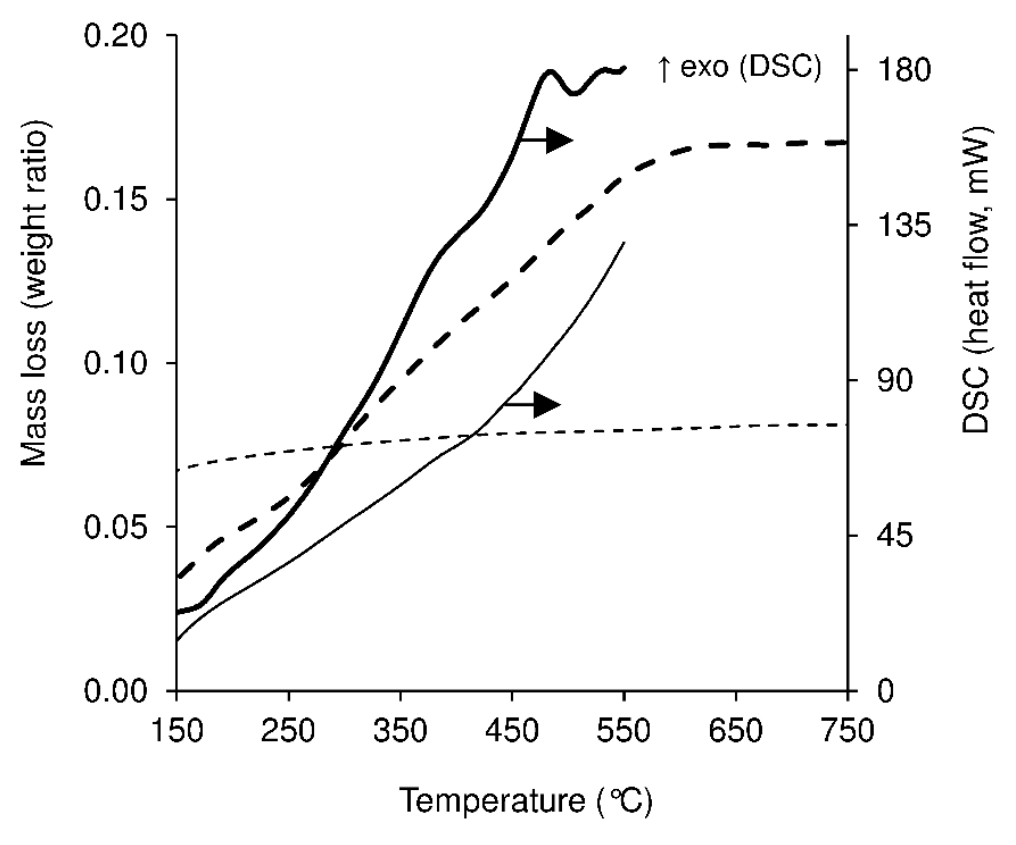

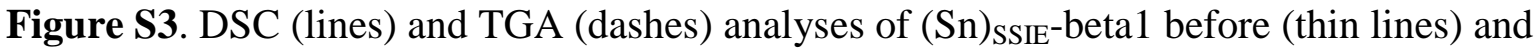
after (thick lines) reaction of Fur in $2 \mathrm{BuOH}$. The used catalyst was washed and dried at 85 ${ }^{\circ} \mathrm{C}$. 